\title{
SEMILINEAR WAVE EQUATIONS
}

\author{
MICHAEL STRUWE
}

\begin{abstract}
We survey existence and regularity results for semi-linear wave equations. In particular, we review the recent regularity results for the $u^{5}$-Klein Gordon equation by Grillakis and this author and give a self-contained, slightly simplified proof.
\end{abstract}

\section{CONTENTS}

\section{Introduction}

2. Preliminaries

3. Rauch's result

4. Large data

5. A remark on the super-critical case

\section{INTRODUCTION}

In this survey we shall be interested in initial value problems for nonlinear wave equations of the type

$$
\begin{array}{cc}
u_{t t}-\Delta u+g(u)=0 & \text { in } \mathbb{R}^{3} \times[0, \infty[, \\
\left.u\right|_{t=0}=u_{0}, & \left.u_{t}\right|_{t=0}=u_{1},
\end{array}
$$

where $g: \mathbb{R} \rightarrow \mathbb{R}$ and the initial data are given sufficiently smooth functions, and $u_{t}=\frac{\partial}{\partial t} u$, etc. The linear case $g(u)=m u$, where $m \in \mathbb{R}$, corresponds to the classical Klein Gordon equation in relativistic particle physics; the constant $m$ may be interpreted as a mass and hence is generally assumed to be nonnegative. In an attempt to model also nonlinear phenomena like quantization, in the 1950s equations of type (1.1) with nonlinearities like

$$
g(u)=m u+u^{3}, \quad m \geq 0,
$$

were proposed as models in relativistic quantum mechanics with local interaction; see for instance Schiff [13] and Segal [14]. Solutions could be real or complex-valued functions. In the latter case it was natural to assume that the nonlinearity commutes with the phase, that is, there holds

$$
g\left(e^{i \varphi} u\right)=e^{i \varphi} g(u) \quad \text { for all } \varphi \in \mathbb{R},
$$

Received by the editors July 11, 1990 and, in revised form, December 7, 1990 .

1980 Mathematics Subject Classification (1985 Revision). Primary 35L05, 35A05, 35-02. 
and hence, in particular, that $g(0)=0$. In this case, $g$ may be expressed

$$
g(u)=u f\left(|u|^{2}\right) \text {, }
$$

which gives the form of equation (1.1) studied, for instance, by Jörgens [8]. Here, for simplicity, and since all important features of our problem already seem to exist in this case, we confine ourselves to the study of real-valued solutions of equation (1.1). To model effects thought to arise in the case, for instance, of spinor fields $u$, the scalar equation (1.1) also has been considered in space dimensions $n \geq 3$; see [14].

Various other models involving nonlinearities $g$ depending also on $u_{t}$ and $\nabla u$, the spatial gradient of $u$, have been studied. The so-called " $\sigma$-model" involves an equation of type (1.1) for vector-valued functions subject to a certain (nonlinear) constraint. ${ }^{1}$ In this case

$$
g(u)=u\left(\left|u_{t}\right|^{2}-|\nabla u|^{2}\right),
$$

and the solution $u=\left(u_{1}, \ldots, u_{n}\right)$ is constrained to satisfy the condition

$$
|u|^{2}=u_{1}^{2}+\cdots+u_{n}^{2}=1
$$

see Shatah [15] for some recent results on this problem and references.

To limit this survey to a reasonable length, however, we restrict our study to nonlinearities depending only on $u$; that is, the semi-linear case. The examples stated previously suggest that we assume that $g(0)=0$ and that $g$ satisfies polynomial growth

$$
|g(u)| \leq C\left(1+|u|^{p-2}\right)|u| \text { for some } p \geq 2, C \in \mathbb{R} .
$$

Moreover, following Strauss [16, Theorem 3.1], we will assume that $g$ satisfies the conditions

$$
G(u) \geq-C|u|^{2} \text { for some } C \in \mathbb{R},
$$

and

$$
|G(u)| /|g(u)| \rightarrow \infty \text { as }|u| \rightarrow \infty,
$$

where $G(u)=\int_{0}^{u} g(v) d v$. Let us briefly motivate the latter two conditions.

First, (1.4) and (1.5) include the linear case (with no sign condition) or, more generally, the case of Lipschitz nonlinearities. Second, in the super-linear case, that is, if $|g(u)| /|u| \rightarrow \infty$ as $|u| \rightarrow \infty$, conditions (1.4), (1.5) should be regarded as a coerciveness condition. In fact, in this case finite propagation speed $\leq 1$ and conservation of energy imply locally uniform a priori bounds in $L^{2}$ for solutions of (1.1) in terms of the initial data; this will be developed in detail in $\S 2$.

By contrast, in the noncoercive case it is easy to construct solutions of (1.1) with smooth initial data that blow up in finite time; for instance, for any $\alpha>0$ the function

solves the equation

$$
u(x, t)=\frac{1}{(1-t)^{\alpha}}
$$

$$
u_{t t}-\Delta u=\alpha(1+\alpha) u|u|^{\frac{2}{\alpha}}
$$

\footnotetext{
${ }^{1}$ In fact, as observed by Shatah and Tahvildar-Zadeh [21], under suitable symmetry assumptions also $\sigma$-models give rise to semilinear wave equations of type (1.1) on $\mathbb{R}^{4} \times \mathbb{R}$.
} 
and blows up at $t=1$. Observe that for $\alpha=\frac{1}{m}, m \in \mathbb{N}$, the right member of this equation is analytic. Modifying the initial data off $\{x ;|x| \leq 2\}$, say, we even obtain a singular solution with $C^{\infty}$-data having compact support. (See John [7] for a blow-up result for a similar equation.) Thus, conditions like (1.3)-(1.5) seem natural if we are interested in global solutions.

The class $(1.3)-(1.5)$ includes the following special cases

$$
g(u)=m u|u|^{q-2}+u|u|^{p-2}, \quad m \geq 0,2 \leq q<p .
$$

As we shall see, for nonlinearities of this kind the answer to the existence problem for (1.1), (1.2) in a striking way depends on the space dimension $n$ and on the exponent $p$. In particular, in the physically interesting case $n=3$, global existence for $p<6$ can be established with relative ease, while the same question for $p>6$ so far has eluded all research attempts. The "critical" case $p=6$ has only recently been settled and a comprehensive account of this result is one of the objectives pursued in this survey.

In fact, the apparent existence of a "critical power" for (1.1) and recent advances on elliptic problems involving critical nonlinearities prompted our interest in the $u^{5}$-Klein Gordon equation. "Critical powers" very often come into play in nonlinear problems through Sobolev embedding. In particular, $p=6$ is the critical power for the Sobolev embedding $H_{\mathrm{loc}}^{1,2}\left(\mathbb{R}^{3}\right) \hookrightarrow L_{\mathrm{loc}}^{p}\left(\mathbb{R}^{3}\right)$. (In $n$ dimensions the critical power for this embedding is $p=\frac{2 n}{n-2}$.) Moreover, they very often arise naturally from the requirements of scale invariance, that is, whenever "intrinsic" notions are involved. A beautiful example of such a problem is the Yamabe problem concerning the existence of conformal metrics with constant scalar curvature on a given (compact) Riemannian manifold. Through the work of Trudinger, Aubin, and-finally-Schoen this problem has now been completely solved and it has become apparent that at the critical power properties like "compactness of the solution set" depend crucially on global aspects of the problem; in this case, on the topological and differentiable structure of the manifold. See Lee and Parker [9] for a recent survey of the Yamabe problem in this journal.

Incidentally, for nonlinear wave equations (or nonlinear Schrödinger equations $i u_{t}-\Delta u+u|u|^{p-2}=0$ ) there appear to be many "critical powers," depending on what aspect of the problem we consider: global existence, scattering theory, ... ; see Strauss $[16$, p. 14f.]. As regards global existence, it remains to be seen whether the critical power represents only a technical barrier or, in fact, defines the dividing line between qualitatively different regimes of behavior of (1.1), (1.2). Through this survey I would like to invite further research on this topic.

We conclude this introduction with a short overview of the existence results in the case of a pure power

$$
u_{t t}-\Delta u+u|u|^{p-2}=0, \quad p>2 .
$$

For more general nonlinearities of type (1.6) similar results hold true. (In contrast, for problems related to scattering, also the lower order terms of $g$ may be decisive.)

The sub-critical case. For $n=3, p<6$ global existence and regularity was established by Jörgens [8] in 1961. Jörgens also was able to show local (small 
time) existence of regular solutions to (1.7), (1.2) for arbitrarily large $p$. Moreover, he was able to reduce the problem of existence of global, regular solutions to (1.1) to (local) estimates of the $L^{\infty}$-norms of solutions.

These results have been generalized to higher dimensions; however, such extensions have been very hard to obtain. While Jörgens' work relies on the classical representation formula for the 3-dimensional wave equation, this method fails in higher dimensions $n>3$. The fundamental solution to the wave equation no longer is positive; moreover, it carries derivatives transverse to the wave cone. Nevertheless, at least for $n \leq 9$, the existence results of Pecher [11], Brenner-von Wahl [2] now cover the full sub-critical range $p<\frac{2 n}{n-2}$. Regular solutions are unique.

Global weak solutions. On the other hand, by a suitable approximation and using energy estimates, for all $p>2, n \geq 3$ it is possible to construct global weak solutions, satisfying (1.7) in a distributional sense; see Segal [14], Lions [10]. In this case, it even suffices to assume that the initial data $u_{0}, u_{1} \in L_{\text {loc }}^{2}\left(\mathbb{R}^{n}\right)$ with $u_{0} \in L_{\text {loc }}^{p}\left(\mathbb{R}^{n}\right)$ and distributional derivative $\nabla u_{0} \in L_{\text {loc }}^{2}\left(\mathbb{R}^{n}\right)$. Energy estimates immediately give uniqueness of weak solutions in case $p \leq \frac{2 n}{n-2}-\frac{2}{n-2}$; see Browder [3]. However, this range is well below the critical Sobolev exponent $p=\frac{2 n}{n-2}$. In order to improve the range of admissable exponents, more sophisticated tools were developed, based, in particular, on the $L^{p}-L^{q}$-estimates for the wave operator by Strichartz [17]; see also Brenner [1]. In their simplest version, these estimates allow to prove uniqueness of solutions to (1.7), (1.2) for $p \leq \frac{2(n+1)}{n-1}$, the Sobolev exponent in $(n+1)$ space dimensions. In fact, uniqueness can be established for $p<\frac{2 n}{n-2}$; see Ginibre-Velo [4]. In this case, moreover, the unique solution can be shown to be "strong," that is, to possess second derivatives in $L^{2}$ and to satisfy the energy identity [4]. Some of these results will be derived in $\S 2$.

The critical case. In dimension $n=3$, global existence of $C^{2}$-solutions in the critical case $p=6$ was first obtained by Rauch [12], assuming the initial energy

$$
E(u(0))=\int_{\mathbb{R}^{3}}\left(\frac{\left|u_{1}\right|^{2}+\left|\nabla u_{0}\right|^{2}}{2}+\frac{|u|^{6}}{6}\right) d x
$$

to be small. His results will be presented in $\S 3$.

In 1987, also for "large" data global $C^{2}$-solutions were shown to exist by this author [18] in the radially symmetric case $u_{0}(x)=u_{0}(|x|), u_{1}(x)=u_{1}(|x|)$. Finally, Grillakis [6] in 1989 was able to remove the latter symmetry assumption, yielding the following result:

Theorem 1.1. For any $u_{0} \in C^{3}\left(\mathbb{R}^{3}\right), u_{1} \in C^{2}\left(\mathbb{R}^{3}\right)$ there exists a unique solution $u \in C^{2}\left(\mathbb{R}^{3} \times[0, \infty[)\right.$ to the Cauchy problem

$$
\begin{gathered}
u_{t t}-\Delta u+u^{5}=0, \\
u_{\mid t=0}=u_{0},\left.\quad u_{t}\right|_{t=0}=u_{1} .
\end{gathered}
$$

In $\S 4$ we present the detailed proof. Related partial regularity results independently have been obtained by Kapitanskii [20] in 1989. Uniqueness holds among $C^{2}$-solutions. The proof procedes via a priori estimates. The classical representation formula crucially enters. It seems unlikely that regularity or 
uniqueness of weak solutions to (1.8), (1.9) can be established in a similar way. Research on the critical case in higher dimensions is in progress; however, to this moment the results on this subject still seem incomplete. Advances in these questions may require eliminating the use of the wave kernel.

The super-critical case. In $\S 5$ we observe that for sufficiently small initial data the existence of global regular solutions, for instance, to the equation

$$
u_{t t}-\Delta u+u^{5}+u|u|^{p-2}=0 \text { in } \mathbb{R}^{3} \times[0, \infty[,
$$

for any $p>2$ can be deduced as a corollary to Rauch's result. Various qualitative properties of solutions in the super-critical case have recently been studied by Zheng [19].

Other open problems concern scattering theory, involving, in particular, decay estimates for solutions of (1.1) (see Ginibre-Velo [5]), or existence and regularity results for initial-boundary value problems.

\section{Preliminaries}

We begin our study of (1.1) with some general comments about local solvability and global continuation of solutions to (1.1), (1.2). An excellent reference for many fundamental results on nonlinear wave equations is Strauss [16]; our treatment of these issues will be somewhat narrower and directed towards our final goal: the critical power. This restricted aim, however, will enable us to make this paper essentially self-contained and to present a lot of material connected with the existence problem for (1.1), (1.2) in detail, introducing the reader to various approaches to this problem and showing their strengths and limitations.

Representation formulas. The representation of solutions to the inhomogeneous wave equation in terms of the fundamental solution and energy estimates form the basis of our solution method. For any $f \in C^{\infty}, u_{0}, u_{1} \in C^{\infty}$ there exists a unique $C^{\infty}$-solution to the Cauchy problem

$$
\begin{gathered}
u_{t t}-\Delta u=f \quad \text { in } \mathbb{R}^{n} \times[0, \infty[, \\
u_{\mid t=0}=u_{0}, \quad u_{t}, \quad=u_{1=0} .
\end{gathered}
$$

If $n=3$, the most interesting case, this solution, in fact, is given by

$$
\begin{aligned}
u(x, t)= & \frac{d}{d t}\left(\frac{1}{4 \pi t} \int_{\partial B_{t}(x)} u_{0}(y) d y\right)+\frac{1}{4 \pi t} \int_{\partial B_{t}(x)} u_{1}(y) d y \\
& +\frac{1}{4 \pi} \int_{0}^{t} \int_{\partial B_{t-s}(x)} \frac{f(y, s)}{t-s} d y d s,
\end{aligned}
$$

where $B_{r}(x)=\left\{y \in \mathbb{R}^{n} ;|x-y|<r\right\}$. From (2.3) we see immediately that information propagates with speed $\leq 1$. In particular, $u(t)$ has compact support for any $t \geq 0$ if this is the case for $u_{0}, u_{1}$, and $f$. However, (2.3) also shows a fundamental weakness of the classical approach: For $u_{0} \in C^{3}, u_{1} \in C^{2}$, $f \in C^{2}$, the solution $u$ will lie in $C^{2}$, only. That is, we encounter a loss of differentiability. In higher dimensions, a representation formula similar to (2.3) holds, however, involving an even larger number (the integer part of $\frac{n}{2}$, resp. $\frac{n-2}{2}$ ) of derivatives of $u_{0}$, resp. of $u_{1}$ and $f$. This makes the representation 
formula appear to be ill-suited for proving existence of solutions for semilinear equations in dimensions $n>3$.

By contrast, no loss of differentiability will occur if instead of pointwise control of the solution we are content with control of integral norms. The basic observation is the following.

Energy inequality. Upon multiplying $(2.1)$ by $u_{t}$ we obtain

$$
\frac{d}{d t}\left(\frac{\left|u_{t}\right|^{2}+|\nabla u|^{2}}{2}\right)-\operatorname{div}\left(u_{t} \nabla u\right)=f u_{t}
$$

where the terms

$$
e_{0}(u)=\frac{\left|u_{t}\right|^{2}+|\nabla u|^{2}}{2}
$$

and $p(u)=u_{t} \nabla u$ may be interpreted as energy and momentum of the solution $u$. Integrating in $x$, if $u(t)$ has compact support, by Hölder's inequality we obtain

$$
\begin{aligned}
\frac{d}{d t} E_{0}(u(t)) & \leq\left(\int_{\mathbb{R}^{n}}|f(\cdot, t)|^{2} d x\right)^{1 / 2}\left(\int_{\mathbb{R}^{n}}\left|u_{t}(\cdot, t)\right|^{2} d x\right)^{1 / 2} \\
& \leq\left(2 E_{0}(u(t))\right)^{1 / 2}\|f(\cdot, t)\|_{L^{2}\left(\mathbb{R}^{n}\right)},
\end{aligned}
$$

where

$$
E_{0}(u(t))=\int_{\mathbb{R}^{n}} e_{0}(u(\cdot, t)) d x=:\|u(t)\|_{0}^{2}
$$

denotes the "energy norm."

Thus

$$
\frac{d}{d t}\|u(t)\|_{0} \leq \frac{1}{\sqrt{2}}\|f(\cdot, t)\|_{L^{2}\left(\mathbb{R}^{n}\right)} \leq\|f(\cdot, t)\|_{L^{2}\left(\mathbb{R}^{n}\right)} .
$$

In particular, if $f=0$, the "energy" $E_{0}$ is conserved.

Various other conservation laws can be obtained by using further multipliers related to symmetries of the wave operator. Very subtle identities and integral estimates in this way have been found; see Strauss [16, Chapter 2] for an overview of results. In particular, in $\S 4$ we will make use of the integral estimate implied by invariance of the wave operator under dilations $(x, t) \mapsto(R x, R t)$ for $R>0$. For our immediate uses, however, the energy inequality will suffice.

So far, (2.4) has been established rigourously only for $C^{\infty}$-data $u_{0}, u_{1}$, and $f$ with spatially compact support. For our next topic it is essential to extend the validity of (2.4) to distribution solutions of (2.1) for finite energy initial data, that is, for $u_{0}, u_{1} \in L^{2}\left(\mathbb{R}^{n}\right)$ with $\nabla u_{0} \in L^{2}\left(\mathbb{R}^{n}\right)$, and functions $f$ belonging to $L^{2}\left(\mathbb{R}^{n} \times[0, T]\right)$ for any $T>0$. To achieve this extension, by density of $C_{0}^{\infty}\left(\mathbb{R}^{n}\right)$ in $L^{2}\left(\mathbb{R}^{n}\right)$ we may approximate data $u_{0}, u_{1}$ as above by functions $u_{0}^{(m)}, u_{1}^{(m)} \in C_{0}^{\infty}$, converging to $u_{0}, u_{1}$ in energy norm as $m \rightarrow \infty$. Similarly, for any $T>0$ we can find smooth, compactly supported functions $f^{(m)}$ converging to $f$ in $L^{2}\left(\mathbb{R}^{n} \times[0, T]\right)$. Let $u^{(m)}$ be the corresponding sequence of solutions to $(2.1),(2.2)$, given by the classical representation formula. Then, applying (2.4) to the difference $v=u^{(m)}-u^{(l)}$ of any two solutions, we see that $u^{(m)}(\cdot, t)$ is a Cauchy sequence in energy norm, uniformly in $t \in[0, T]$. The 
limit $u$ is a distribution solution to $(2.1),(2.2)$ with uniformly finite energy in the interval $[0, T]$, which satisfies $(2.4)$ in the slightly weaker sense

$$
\|u(t)\|_{0} \leq\|u(0)\|_{0}+\int_{0}^{t}\|f(\cdot, s)\|_{L^{2}\left(\mathbb{R}^{n}\right)} d s,
$$

for all $t \leq T$. In particular, $u$ is unique in this class.

In a similar way, we now use (2.5) to construct solutions to nonlinear wave equations (1.1), (1.2) for smooth, compactly supported initial data and smooth nonlinearities satisfying a global Lipschitz condition by a contraction mapping argument.

Global solutions for Lipschitz nonlinearities. Indeed, if $g: \mathbb{R} \rightarrow \mathbb{R}$ is smooth and globally Lipschitz, for any $v \in C_{0}^{\infty}\left(\mathbb{R}^{n} \times\left[0, \infty[)\right.\right.$ we obtain a $C^{\infty}$-solution $u=K(v)$ to the initial value problem

$$
u_{t t}-\Delta u=-g(v)
$$

with data $u_{0}, u_{1}$. By (2.5), for all $T>0$ we have

$$
\begin{aligned}
\sup _{0 \leq t \leq T}\|(K(v)-K(\tilde{v}))(t)\|_{0} & \leq \int_{0}^{T}\|(g(v)-g(\tilde{v}))(t)\|_{L^{2}} d t \\
& \leq L \int_{0}^{T}\|(v-\tilde{v})(t)\|_{L^{2}} d t \\
& \leq T L \sup _{0 \leq t \leq T}\|(v-\tilde{v})(t)\|_{L^{2}},
\end{aligned}
$$

where $L$ denotes the Lipschitz constant of $g$. Moreover, if $u_{0}, u_{1}$ have support in $B_{R}(0)$, and if $v(t)$ has support in $B_{R+t}(0)$, so will $u(t)$. Finally, by Poincaré's inequality, for such $v, \tilde{v}$, and $t \leq 1$ we can estimate

$$
\|(v-\tilde{v})(t)\|_{L^{2}} \leq(R+t)\|\nabla(v-\tilde{v})(t)\|_{L^{2}} \leq \sqrt{2}(R+1)\|(v-\tilde{v})(t)\|_{0} .
$$

Thus, for $T \leq \min \left\{1, \frac{1}{\sqrt{2} L(R+1)}\right\}$ the map $K$ extends to a contracting map on the space

$$
V=\left\{v \in L^{2}\left(\mathbb{R}^{n} \times[0, T]\right) ; \operatorname{supp}(v(t)) \subset B_{R+t}(0), v_{t}(t), \nabla v(t) \in L^{2}\left(\mathbb{R}^{n}\right)\right.
$$

for almost every $t$, and $\left.\sup _{0 \leq t \leq T}\|v(t)\|_{0}<\infty\right\}$,

endowed with the norm

$$
\|v\|_{V}=\sup _{0 \leq t \leq T}\|v(t)\|_{0} .
$$

Let $u$ be the unique fixed point of $K$ in $V$; then $u$ weakly solves (1.1) and assumes its initial data (1.2) in the distribution sense. By an approximation argument as in the preceding paragraph, likewise for compactly supported measurable initial data with finite energy we obtain a (unique) local solution to (1.1), (1.2) in the space $V$. Observe that the support of the solution grows with speed $\leq 1$. Hence, given any number $T_{0}>0$, by iterating the above construction a finite number of times (with $T \leq \min \left\{1, \frac{1}{\sqrt{2} L\left(R+T_{0}\right)}\right\}$ ) we obtain 
a finite energy solution to $(1.1),(1.2)$ on the interval $\left[0, T_{0}\right]$ for any finite energy initial data supported in a ball of radius $R$. Since $T_{0}$ is arbitrary, this solution can be continued globally.

Finally, by finiteness of propagation speed also the assumption that the initial data be compactly supported can be removed. Indeed, if $u$ and $v$ solve (1.1) for compactly supported, finite energy initial data that coincide on the ball $B_{R}(0)$, their difference $\tilde{u}$ will solve an equation of type (1.1) with a Lipschitz nonlinearity $\tilde{g}$, where $\tilde{g}(\tilde{u})=g(u)-g(v)$, and initial data vanishing on $B_{R}(0)$. By the above existence and uniqueness result, $\tilde{u}$ is supported outside the light cone $K=\{(x, t) ;|x|<R-t\}$ above $B_{R}(0)$. Hence a solution to (1.1) on $K$ is entirely determined by its data in $B_{R}(0)$. For arbitrary data $u_{0}, u_{1}$ with locally finite energy, and $k \in \mathbb{N}$, we then let $u_{0}^{(k)}, u_{1}^{(k)}$ be compactly supported data that agree with $u_{0}, u_{1}$ on $B_{k}(0)$. For any $k \in \mathbb{N}$ the corresponding global solutions $u^{(k)}, k \geq k_{0}$, then agree on the cone above $B_{k_{0}}(0)$. Hence the sequence $\left(u^{(k)}\right)$ converges locally in energy norm to a global solution $u$ of (1.1), (1.2). In the same way, as far as global existence is concerned, in the following for convenience-and with no loss of generality-we may suppose that the initial data have compact support. Moreover, for our next topics we also require the data $u_{0}, u_{1}$ to be smooth.

Strong solutions. Taking difference quotients $u^{(h)}=\frac{u(\cdot)-u(\cdot+h e)}{h}$ in any space direction $e$ and passing to the limit $h \rightarrow 0$ we see that $v=e \cdot \nabla u$ weakly solves

$$
v_{t t}-\Delta v+g^{\prime}(u) v=0
$$

and satisfies

$$
\begin{aligned}
\|v(T)\|_{0}-\|v(0)\|_{0} & \leq \int_{0}^{T}\left\|\left(g^{\prime}(u) v\right)(t)\right\|_{L^{2}} d t \leq L \int_{0}^{T}\|v(t)\|_{L^{2}} d t \\
& \leq C L \int_{0}^{T}\|v(t)\|_{0} d t
\end{aligned}
$$

for any $T>0$. Thus $\nabla u_{t}(t), \nabla^{2} u(t) \in L^{2}\left(\mathbb{R}^{n}\right)$, uniformly in $t \in[0, T]$, and from equation (1.1) it now also follows that $u_{t t}(t) \in L^{2}$, uniformly in $t \in[0, T]$, for any $T>0$. This is the class of "strong solutions" to nonlinear wave equations. For strong solutions we can derive the strong form of the energy inequality (2.4). Since $g(u) u_{t}=\frac{d}{d t} G(u)$, upon multiplying (1.1) by $u_{t}$ we obtain the conservation law

$$
\frac{d}{d t}\left(\frac{\left|u_{t}\right|^{2}+|\nabla u|^{2}}{2}+G(u)\right)-\operatorname{div}\left(u_{t} \nabla u\right)=0,
$$

where the term

$$
e(u(t))=\frac{\left|u_{t}\right|^{2}+|\nabla u|^{2}}{2}+G(u)=e_{0}(u)+G(u)
$$

now also contains the "potential energy density" $G(u)$. Let

$$
E(u(t))=\int_{\mathbb{R}^{n}} e(u(t)) d x .
$$


Integrating over $\mathbb{R}^{n}$, since $u(t)$ has compact support, we thus obtain that

$$
\frac{d}{d t} E(u(t))=0,
$$

and energy is strictly conserved.

Higher regularity. By iterating the above procedure, we may want to derive $L^{2}$-bounds for higher and higher derivatives $D^{k} u$, where $D$ denotes any spacetime derivative, $k \in \mathbb{N}_{0}$. For instance, in case $k=3$, any second order spatial derivative $w=\nabla^{2} u$ satisfies

$$
w_{t t}-\Delta w+g^{\prime}(u) w+g^{\prime \prime}(u)|\nabla u|^{2}=0 .
$$

However, while the term involving $g^{\prime}(u) w$ can be dealt with as before, the second term presents some difficulty and can only be controlled in terms of $E_{0}(w)$ if the dimension $n \leq 8$. In this case, assuming $\left|g^{\prime \prime}(u)\right| \leq C$, by Sobolev's inequality we can estimate

$\left\|g^{\prime \prime}(u)|\nabla u(t)|^{2}\right\|_{L^{2}} \leq C\|\nabla u(t)\|_{L^{4}}^{2} \leq C|| \nabla^{2} u(t)\left\|_{L^{2}}^{2 \gamma}\right\| \nabla^{3} u(t) \|_{L^{2}}^{2-2 \gamma} \leq C E_{0}(w)^{1-\gamma}$

where $\frac{\gamma}{\frac{2 n}{n-2}}+\frac{(1-\gamma)}{\frac{2 n}{n-4}} \leq \frac{1}{4}$. The energy inequality (2.4) formally yields

$$
\frac{d}{d t}\|w(t)\|_{0} \leq C\|w(t)\|_{0}+C\|w(t)\|_{0}^{2-2 \gamma} .
$$

Note that $\gamma \rightarrow 0$ as $n \nearrow 8$. Hence the last exponent may be $>1$, and $\|w(t)\|_{0}$ might blow up in finite time. Similar problems arise if we want to control higher derivatives of $u$ by this simple trick.

If $n \geq 9$, we cannot start our iteration at $k_{0}=2$. However, if we choose $k_{0} \in \mathbb{N}$ sufficiently large, by using Sobolev's embedding theorem as above we can derive a priori estimates for $\left\|D^{k} u(t)\right\|_{0}$ for any $k \geq k_{0}$ in a small time interval $0 \leq t \leq T(k)$. As in low dimensions, these estimates also may blow up in finite time.

Nevertheless, we can use these estimates to show the local (small time) existence of solutions to general semilinear equations (1.1) for smooth, compactly supported initial data.

Local solutions for semi-linear equations. Indeed, given an arbitrary smooth map $g: \mathbb{R} \rightarrow \mathbb{R}$ we may approximate $g$ by maps $g^{(k)}$ satisfying a uniform Lipschitz condition and coinciding with $g$ for $|u| \leq k$.

By the preceding discussion, given any smooth initial data of compact support, for each $k \in \mathbb{N}$ we obtain a global strong solution $u^{(k)}$ of the approximate equation

$$
u_{t t}-\Delta u+g^{(k)}(u)=0,
$$

with $\left\|D^{l} u^{(k)}(t)\right\|_{0} \leq C$ for any $l \in\left\{0, \ldots, l_{0}\right\}$ on some interval $0 \leq t \leq T=$ $T\left(l_{0}\right)$, where $C$ depends on the Lipschitz constant of $g^{(k)}, l_{0}, T$, and the size of the support of the initial data.

If $n=3$, by the Sobolev embedding theorem

$$
\left\|D^{l} u^{(k)}(t)\right\|_{L^{\infty}} \leq C\left\|D^{2+l} u^{(k)}(t)\right\|_{L^{2}} \leq C\left\|D^{1+l} u^{(k)}(t)\right\|_{0},
$$


for $l=0,1,2$. In particular, for large $k \in \mathbb{N}$ and sufficiently small $T>0$, we obtain $\left|u^{(k)}(x, t)\right| \leq k$ in $\mathbb{R}^{3} \times[0, T]$, and $u^{(k)}$ will be a solution to (1.1). Similarly, if $n>3$ we can bound

$$
\left\|D^{l} u^{(k)}(t)\right\|_{L^{\infty}}
$$

in terms of $\left\|D^{m+l} u^{(k)}(t)\right\|_{0}$, where $m>\frac{n}{2}-1$, and we may conclude as before.

Again remark that by finiteness of propagation speed the assumption that the initial data be compactly supported can be removed; in this case, however, we can only assert the existence of a solution to (1.1), (1.2) in a neighborhood of $\mathbb{R}^{n} \times\{0\}$.

Global weak solutions. We now specialize our nonlinearity $g$ to be of the form (1.3)-(1.5). By assumption (1.4) there exist sequences $r_{k}^{ \pm} \rightarrow \pm \infty$ as $k \rightarrow \infty$ such that

$$
r_{k}^{ \pm} g\left(r_{k}^{ \pm}\right) \geq-C\left|r_{k}^{ \pm}\right|^{2}
$$

We approximate $g$ by Lipschitz functions

$$
g^{(k)}(u)= \begin{cases}g\left(r_{k}^{-}\right), & \text {if } u<r_{k}^{-}, \\ g(u), & \text { if } r_{k}^{-} \leq u \leq r_{k}^{+}, \\ g\left(r_{k}^{+}\right), & \text {if } u>r_{k}^{+},\end{cases}
$$

with primitive $G^{(k)}(u)$. Note that the approximating functions $g^{(k)}$ satisfy (1.4) with a uniform constant $C$. Now, for any $k \in \mathbb{N}$ and smooth, compactly supported data we obtain a unique global strong solution $u^{(k)}$ to the approximate problem (1.1), (1.2) with $D^{2} u^{(k)}(t) \in L^{2}\left(\mathbb{R}^{n}\right)$ for all $t$.

Conservation of energy (2.7) implies uniform bounds for $u=u^{(k)}$. Let

$$
E^{(k)}(u(t))=E_{0}(u(t))+\int_{\mathbb{R}^{n}} G^{(k)}(u(t)) d x
$$

By (1.4), (2.7), for any $t \geq 0$ we have

$$
E_{0}(u(t))-C\|u(t)\|_{L^{2}}^{2} \leq E^{(k)}(u(t))=E^{(k)}(u(0)) \leq C<\infty,
$$

uniformly in $k \in \mathbb{N}$. In order to control $\|u(t)\|_{L^{2}}$, for fixed $x \in \mathbb{R}^{n}$ we estimate

$$
\left|u(x, t)-u_{0}(x)\right|^{2}=\left|\int_{0}^{t} u_{t}(x, s) d s\right|^{2} \leq t \int_{0}^{t}\left|u_{t}(x, s)\right|^{2} d s .
$$

Integrating in $x$, by Minkowski's inequality we obtain

$$
\|u(t)\|_{L^{2}} \leq\left\|u_{0}\right\|_{L^{2}}+\left(2 t \int_{0}^{t} E_{0}(u(s)) d s\right)^{1 / 2} .
$$

For $t \leq T$ this and (2.8) gives the integral inequality

$$
E_{0}(u(t)) \leq C+C T \int_{0}^{t} E_{0}(u(s)) d s
$$

for $E_{0}(u(t))$. From Gronwall's lemma we thus conclude that $u(t)=u^{(k)}(t)$ is uniformly bounded in energy norm on any interval $[0, T]$, uniformly in $k \in \mathbb{N}$.

Hence, $\left(u^{(k)}\right)_{k \in \mathbb{N}}$ is weakly relatively compact in the energy norm. Moreover, the support of $u^{(k)}(t)$ is bounded uniformly in $k$, for all $t \leq T$. By the RellichKondrakov theorem, therefore, we may assume that $u^{(k)} \rightarrow u$ strongly in $L^{2}(Q)$ 
on any compact space-time region $Q$ and pointwise almost everywhere. The limit $u$ has finite energy

$$
E_{0}(u(t)) \leq \liminf _{k \rightarrow \infty} E_{0}\left(u^{(k)}(t)\right)
$$

and

$$
\int_{\mathbb{R}^{n}} G(u(t)) d x \leq \liminf _{k \rightarrow \infty} \int_{\mathbb{R}^{n}} G^{(k)}\left(u^{(k)}(t)\right) d x
$$

for almost every $t>0$, by Fatou's lemma. Finally, for $\varphi \in C_{0}^{\infty}\left(\mathbb{R}^{n} \times\right] 0, \infty[)$ we obtain

$$
\begin{aligned}
\iint\left(u_{t} \varphi_{t}-\nabla u \nabla \varphi\right) d x d t & =\lim _{k \rightarrow \infty} \iint\left(u_{t}^{(k)} \varphi_{t}-\nabla u^{(k)} \nabla \varphi\right) d x d t \\
& =\lim _{k \rightarrow \infty} \iint g^{(k)}\left(u^{(k)}\right) \varphi d x d t=\iint g(u) \varphi d x d t
\end{aligned}
$$

where $\iint \ldots$ denotes integration over $\mathbb{R}^{n} \times[0, T]$. That is, $u$ weakly solves equation (1.1). (Vitali's theorem, (1.5) and (2.9) were used to pass to the limit in the nonlinear term.) Similarly, approximating $L^{2}$-data $u_{0}, u_{1}$ of finite energy

$$
\int_{\mathbb{R}^{n}}\left(\frac{\left|u_{1}\right|^{2}+\left|\nabla u_{0}\right|^{2}}{2}+G\left(u_{0}\right)\right) d x<\infty
$$

by functions $u_{0}^{(k)}, u_{1}^{(k)} \in C_{0}^{\infty}\left(\mathbb{R}^{n}\right)$, the existence of global weak solutions to (1.1) for arbitrary finite energy data may be derived.

Regularity and uniqueness. In the special case (1.7) with $p \leq \frac{2 n}{n-2}-\frac{2}{n-2}$ energy estimates may be used to obtain higher regularity and uniqueness. Indeed, let $u^{(h)}$ be a difference quotient in direction $e \in \mathbb{R}^{n}$ as before. Then, upon passing to the limit $h \searrow 0$, for $v=e \cdot \nabla u$ we obtain

$$
v_{t t}-\Delta v=(1-p)|u|^{p-2} v,
$$

and thus, formally, by Hölder's inequality and (2.4), that

$$
\frac{d}{d t}\|v(t)\|_{0} \leq C\left\|\left(|u|^{p-2} v\right)(t)\right\|_{L^{2}} \leq C\|u(t)\|_{L^{2^{*}}}^{p-2}\|v(t)\|_{L^{2^{*}}},
$$

where $2^{*}=\frac{2 n}{n-2}$. Sobolev's inequality now implies that

$$
\frac{d}{d t}\|v(t)\|_{0} \leq C\|u(t)\|_{0}^{p-2}\|v(t)\|_{0}
$$

and it follows that $E_{0}(v(t))<\infty$ for all $t$; that is, $u$ is a strong solution to (1.1). Similarly, higher regularity (for small time, if the dimension is large) may be obtained. To see uniqueness, let $u, \tilde{u}$ be solutions to (1.7) with the same initial data (1.2). For $v=u-\tilde{u}$ we obtain the inequality

$$
\frac{d}{d t}\|v(t)\|_{0} \leq C\left(\|u(t)\|_{0}+\|\tilde{u}(t)\|_{0}\right)^{p-2}\|v(t)\|_{0}
$$

Since $v(0)=v_{t}(0)=0$, uniqueness follows.

By more sophisticated methods the above regularity and uniqueness results may be extended to the full sub-critical range $p<\frac{2 n}{n-2}$; see Ginibre-Velo [4]. One such method will be briefly explained next. 
$L^{p}-L^{q}$-estimates. By a result of Strichartz [17], for any $L^{p}$-solution of the wave equation in $\mathbb{R}^{n} \times\left[0, \infty\left[\right.\right.$ with $\square u=u_{t t}-\Delta u \in L^{\frac{p}{p-1}}$ and vanishing initial data we have

$$
\|u(t)\|_{L^{p}} \leq C \int_{0}^{t}(t-s)^{1-2 n \delta}\|\square u(s)\|_{L^{\frac{p}{p-1}}} d s,
$$

provided $\delta=\frac{1}{2}-\frac{1}{p} \leq \frac{1}{n+1}$; see also Brenner [1]. We illustrate how this estimate may be used to obtain global strong (or even classical) solutions to (1.7) in dimensions $n>3$. Since we will need $g \in C^{2}$, we suppose that $p \geq 3$. The above condition on $\delta$ then requires $n \leq 5, p \leq \frac{2(n+1)}{n-1}$.

It suffices to show existence of a solution on $\mathbb{R}^{n} \times[0, T]$ for compactly supported, smooth data and for arbitrary $T>0$. Let $u^{(0)}$ solve $u_{t t}^{(0)}-\Delta u^{(0)}=0$ with initial data (1.2), and for $k \in \mathbb{N}$ let $u^{(k)}$ be the solution to the approximate equation

$$
u_{t t}^{(k)}-\Delta u^{(k)}+u^{(k)} \min \left\{\left|u^{(k)}\right|^{p-2}, k^{p-2}\right\}=0,
$$

together with (1.2). Here, $\min \{a, b\}$ is a smooth function coinciding with the minimum of $a$ and $b$ for $|a-b| \geq 1$.

Then $u^{(k)}=u^{(0)}+v^{(k)}$, where

$$
\square v^{(k)}=v_{t t}^{(k)}-\Delta v^{(k)}=-u^{(k)} \min \left\{\left|u^{(k)}\right|^{p-2}, k^{p-2}\right\},
$$

with

$$
v^{(k)}(0)=v_{t}^{(k)}(0)=0
$$

By (2.6), $D^{2} u^{(k)}(t) \in L^{2}\left(\mathbb{R}^{n}\right)$ for all $t$. Moreover, by (2.9) we can uniformly bound

$$
\begin{gathered}
E_{0}\left(u^{(k)}(t)\right) \leq C_{0}, \\
\int_{\mathbb{R}^{n}} \min \left\{\frac{\left|u^{(k)}\right|^{p}}{p}, \frac{k^{p}}{p}+\frac{\left(\left|u^{(k)}\right|^{2}-k^{2}\right) k^{p-2}}{2}\right\} d x \leq C_{0} .
\end{gathered}
$$

That is, $\square v^{(k)}(t)$ is uniformly bounded in $L^{\frac{p}{p-1}}\left(\mathbb{R}^{n}\right)$ for all $t$. From (2.10) we now obtain

$$
\begin{aligned}
\left\|u^{(k)}(t)\right\|_{L^{p}} & \leq\left\|u^{(0)}(t)\right\|_{L^{p}}+\left\|v^{(k)}(t)\right\|_{L^{p}} \\
& \leq C(t)+C \int_{0}^{t}(t-s)^{1-2 n \delta}\left\|\square v^{(k)}(s)\right\|_{L^{p-1}} d x \leq C(T)
\end{aligned}
$$

for all $t<T$, since $\delta=\frac{1}{2}-\frac{1}{p} \leq \frac{1}{n+1}<\frac{1}{n}$ for the range of $p$ and $n$ considered.

Differentiating (2.11), similarly we obtain

$$
\left|\square\left(D v^{(k)}\right)\right| \leq(p-1)\left|D u^{(k)}\right| \min \left\{\left|u^{(k)}\right|^{p-2}, k^{p-2}\right\} \leq(p-1)\left|D u^{(k)}\right|\left|u^{(k)}\right|^{p-2} .
$$

Hence $\square\left(D v^{(k)}\right) \in L^{\frac{p}{p-1}}$ and by $(2.12)$

$$
\left\|\square\left(D v^{(k)}\right)(t)\right\|_{L^{\frac{p}{p-1}}} \leq(p-1)\left\|D u^{(k)}(t)\right\|_{L^{p}}\left\|u^{(k)}(t)\right\|_{L^{p}}^{p-2} \leq C(T)\left\|D u^{(k)}(t)\right\|_{L^{p}} .
$$

Thus, from (2.10) we infer

$$
\left\|D u^{(k)}(t)\right\|_{L^{p}} \leq C(T)+C(T) \int_{0}^{t}(t-s)^{1-2 n \delta}\left\|D u^{(k)}(s)\right\|_{L^{p}} d s,
$$

and it follows that $\left\|D u^{(k)}(t)\right\|_{L^{p}} \leq C(T)$ for all $0 \leq t \leq T$. 
Finally, we have

$$
\left|\square\left(D^{2} v^{(k)}\right)\right| \leq C\left(\left|D u^{(k)}\right|^{2}\left|u^{(k)}\right|^{p-3}+\left|D^{2} u^{(k)}\right|\left|u^{(k)}\right|^{p-2}\right),
$$

whence

$$
\begin{aligned}
\left\|\square\left(D^{2} v^{(k)}\right)(t)\right\|_{L^{\frac{p}{p-1}}} & \leq C\left\|D u^{(k)}(t)\right\|_{L^{p}}^{2}\left\|u^{(k)}(t)\right\|_{L^{p}}^{p-3}+C\left\|D^{2} u^{(k)}(t)\right\|_{L^{p}}\left\|u^{(k)}(t)\right\|_{L^{p}}^{p-2} \\
& \leq C(T)\left(1+\left\|D^{2} u^{(k)}(t)\right\|_{L^{p}}\right)
\end{aligned}
$$

and from (2.10) again it follows that $\left\|D^{2} u(k)(t)\right\|_{L^{p}} \leq C(T)$, uniformly in $k$. But by Sobolev's inequality, for $n \leq 5, p \geq 3$, we may estimate

$$
\left\|u^{(k)}(t)\right\|_{L^{\infty}} \leq C\left\|D^{2} u^{(k)}(t)\right\|_{L^{p}} \leq C(T) .
$$

It follows that for sufficiently large $k$ the function $u=u^{(k)}$ is a (strong) solution to the original equation (1.7). If $g \in C^{4}$, we can proceed to bound the first and second derivatives of $u$ and hence obtain a classical solution.

Note that the range $p \leq \frac{2 n+2}{n-1}$, where Stichartz' estimate may be applied, slightly exceeds the range $p \leq \frac{2 n}{n-2}-\frac{2}{n-2}$, where simple energy estimates suffice to show regularity and uniqueness.

Classical solutions. If $n=3$, using (2.3) one can also devise a contraction mapping argument in the space $C^{2}$ to obtain local classical solutions to (1.1), (1.2) for initial data $u_{0} \in C^{3}, u_{1} \in C^{2}$ with compact support.

Indeed, via (2.3) the initial value problem (1.1), (1.2) can be converted into the integral equation

$$
u(x, t)=v(x, t)-\frac{1}{4 \pi} \int_{0}^{t} \int_{|x-y|=t-s} \frac{g(u(y, s))}{t-s} d y d s,
$$

where $v$ denotes the solution to the homogeneous wave equation with data (1.2). If $g$ is smooth and globally Lipschitz this can easily be solved on $\mathbb{R}^{3} \times[0, T]$ for suitably small $T>0$ by a contraction mapping argument in the space $C^{0}\left(\mathbb{R}^{3} \times[0, T]\right)$ with the $L^{\infty}$-norm. Differentiating $(1.1)$ in any spatial direction, similarly we obtain

$$
D u(x, t)=D v(x, t)-\frac{1}{4 \pi} \int_{0}^{t} \int_{|x-y|=t-s} \frac{g^{\prime}(u) D u}{t-s} d y d s
$$

and an analogous equation for the second spatial derivatives, from which we can as usual derive locally uniform bounds for all first and second derivatives of $u$ on $\mathbb{R}^{3} \times[0, T]$. To extend $u$ beyond $T$ we write

$$
u(x, t)=v_{1}(x, t)-\frac{1}{4 \pi} \int_{T}^{t} \int_{|x-y|=t-s} \frac{g(u(y, s))}{t-s} d y d s,
$$

where now $v_{1}$ denotes the solution of the homogeneous wave equation with data $u(\cdot, T)$ and $u_{t}(\cdot, T)$ at time $T$. At first it may seem as if we had lost one derivative in this procedure. However, following Jörgens $[8$, p. 301], we can write

$$
v_{1}(x, t)=v(x, t)-\frac{1}{4 \pi} \int_{0}^{T} \int_{|x-y|=t-s} \frac{g(u(y, s))}{t-s} d y d s,
$$


and $v_{1} \in C^{2}$, as desired. Thus, for smooth Lipschitz nonlinearities by iteration we obtain global $C^{2}$-solutions. Likewise, for smooth $g$ we obtain local $C^{2}$-solutions (for small time). However, if $g^{\prime}(u)$ is uniformly bounded (for instance, if $u$ is uniformly bounded) on any interval $[0, T]$, then also this solution extends globally. Finally, by finiteness of propagation speed, the assumption that the data have compact support can be dropped.

Due to loss of differentiability in the nonlinear term, in dimensions $n>3$ this approach-apparently-fails.

After this preliminary discussion of different approaches to semi-linear wave equations we now focus our attention on (1.7) in the critical case $p=6$ in dimension $n=3$, which will be fixed from now on.

In the next section we present the existence result of Rauch for small data. Then we present an energy decay estimate and show how regularity in the radial case may be derived. Finally we focus on the work of Grillakis [6], whose penetrating analysis provides the crucial insight needed to pass from the radially symmetric to the general case and give a slightly simplified exposition. We conclude this paper with a global existence result for certain super-critical nonlinearities and small data.

\section{RAUCH'S RESULT}

Let $z=(x, t)$ denote points in space-time $\mathbb{R}^{3} \times \mathbb{R}$. Given $z_{0}=\left(x_{0}, t_{0}\right)$, let

$$
K\left(z_{0}\right)=\left\{z=(x, t) ;\left|x-x_{0}\right| \leq t_{0}-t\right\}
$$

be the backward light cone with vertex at $z_{0}$,

$$
M\left(z_{0}\right)=\left\{z=(x, t) ;\left|x-x_{0}\right|=t_{0}-t\right\}
$$

its mantle,

$$
D\left(t ; z_{0}\right)=\left\{z=(x, t) \in K\left(z_{0}\right)\right\} \quad(t \text { fixed })
$$

its space-like sections. If $z_{0}=(0,0), z_{0}$ will be omitted. For any space-time region $Q \subset \mathbb{R}^{3} \times \mathbb{R}, T<S$, we denote

$$
Q_{T}^{S}=\{z=(x, t) \in Q ; T \leq t \leq S\}
$$

the trunctated region. Hence, for instance,

$$
\partial K_{t}^{s}=D(s) \cup D(t) \cup M_{t}^{s} .
$$

If $s=\infty$ or $t=-\infty$, they will be omitted. Given a function $u$ on a cone $K\left(z_{0}\right)$, we denote its energy density by

$$
e(u)=\frac{1}{2}\left(\left|u_{t}\right|^{2}+|\nabla u|^{2}\right)+\frac{1}{6}|u|^{6},
$$

and by

$$
E\left(u ; D\left(t ; z_{0}\right)\right)=\int_{D\left(t ; z_{0}\right)} e(u) d x
$$

its energy on the space-like section $D\left(t ; z_{0}\right)$. Moreover, let $x=y+x_{0}$ and denote

$$
d_{z_{0}}(u)=\frac{1}{2}\left|\frac{y}{|y|} u_{t}-\nabla u\right|^{2}+\frac{1}{6}|u|^{6}
$$


the energy density of $u$ tangent to $M\left(z_{0}\right)$.

Finally, for $x_{0} \in \mathbb{R}^{3}$ let

$$
B_{R}\left(x_{0}\right)=\left\{x \in \mathbb{R}^{3} ;\left|x-x_{0}\right|<R\right\}
$$

with boundary

$$
S_{R}\left(x_{0}\right)=\left\{x \in \mathbb{R}^{3} ;\left|x-x_{0}\right|=R\right\} .
$$

In the following, the letters $c, C$ will denote various constants. $E_{0}$ will denote a bound for the initial energy.

The proof of Rauch's existence result relies on the following inequalities of Hardy-type that also play an essential role in the work of Grillakis and this author on the limit case $p=6$. We state these estimates in a form due to Grillakis [6, Lemma 2.1]).

Lemma 3.1. Suppose $u \in L^{6}\left(B_{R}\right)$ possesses a weak radial derivative $u_{r}=\frac{x \cdot \nabla u}{|x|} \in$ $L^{2}\left(B_{R}\right)$. Then with an absolute constant $C_{0}$ for all $0 \leq \rho<R$ the following holds:

$$
\begin{aligned}
& \int_{B_{R} \backslash B_{\rho}} \frac{|u(x)|^{2}}{|x|^{2}} d x \leq 4 \int_{B_{R} \backslash B_{\rho}}\left|u_{r}\right|^{2} d x+2 R^{-1} \int_{S_{R}}|u|^{2} d o \\
& \int_{B_{R}} \frac{|u(x)|^{2}}{|x|^{2}} d x \leq C_{0}\left(\int_{B_{R}}\left|u_{r}\right|^{2} d x+\left(\int_{B_{R}}|u|^{6} d x\right)^{1 / 3}\right) ;
\end{aligned}
$$

$$
\int_{S_{R}}|u|^{4} d o \leq C_{0}\left(\left(\int_{B_{R}}\left|u_{r}\right|^{2} d x\right)^{1 / 2}\left(\int_{B_{R}}|u|^{6} d x\right)^{1 / 2}+\left(\int_{B_{R}}|u|^{6} d x\right)^{2 / 3}\right) .
$$

Proof. (i) follows upon integrating the inequality

$$
\left|u_{r}\right|^{2}=\left|\frac{1}{\sqrt{r}} \partial_{r}(\sqrt{r} u)-\frac{1}{2 r} u\right|^{2} \geq \frac{|u|^{2}}{4 r^{2}}-\frac{1}{2 r^{2}} \frac{\partial}{\partial r}\left(r u^{2}\right)
$$

over $B_{R} \backslash B_{\rho}$. See Grillakis [6, Lemma 2.1] for the remaining details of the proof.

Let $z_{0}=\left(x_{0}, t_{0}\right)$ be given and suppose $u$ is a $C^{2}$-solution of (1.8) on the backward light cone $K_{0}\left(z_{0}\right)$. As observed in $\S 2$ above, in order to prove that $u$ can be extended to a global solution of (1.8), it suffices to show that for any $z_{0}$ as above

$$
m_{0}=\sup _{K_{0}\left(z_{0}\right)}|u|
$$

can be a priori bounded in terms of $z_{0}$ and the initial data. Clearly, we may assume that $m_{0}=\left|u\left(z_{0}\right)\right|$.

The first fundamental estimate towards deriving a priori bounds of this kind is the following local version of the energy inequality. For later use we refer to a slightly more general situation than described above.

Lemma 3.2. Let $\bar{z}=(\bar{x}, \bar{t})$. Suppose $u \in C^{2}\left(K_{0}(\bar{z}) \backslash\{\bar{z}\}\right)$ solves (1.8), (1.9). Then for any $0 \leq t<s<\bar{t}$ there holds

$$
E(u ; D(s ; \bar{z}))+\frac{1}{\sqrt{2}} \int_{M_{l}^{s}(\bar{z})} d_{\bar{z}}(u) d o=E(u ; D(t ; \bar{z})) \leq E_{0},
$$


where do denotes the surface measure on $M(\bar{z})$.

Proof. Integrate the identity

$$
\left(u_{t t}-\Delta u+u^{5}\right) u_{t}=\frac{d}{d t} e(u)-\operatorname{div}\left(\nabla u u_{t}\right)=0
$$

over $K_{t}^{s}(\bar{z})$. Now let $y=x-\bar{x}$ and use the fact that the outward unit normal on $M(\bar{z})$ is given by

$$
n=\frac{1}{\sqrt{2}}\left(\frac{y}{|y|}, 1\right) \text {. }
$$

Hence the "energy flux" through $M(\bar{z})$ is given by

$$
\begin{aligned}
n \cdot\left(-\nabla u u_{t}, e(u)\right) & =\frac{1}{\sqrt{2}}\left(\frac{1}{2}\left(\left|\partial_{t} u\right|^{2}-2 \frac{y}{|y|} \cdot \nabla u u_{t}+|\nabla u|^{2}\right)+\frac{1}{6}|u|^{6}\right) \\
& =\frac{1}{\sqrt{2}} d_{\bar{z}}(u) .
\end{aligned}
$$

See Rauch [12].

By Lemma 3.2, for any fixed $\bar{z}$ the energy $E(u ; D(s ; \bar{z}))$ is a monotone nonincreasing function of $s \in[0, \bar{t}$ and hence converges to a limit as $s \nearrow \bar{t}$. It follows that

$$
\int_{M_{t}^{s}(\bar{z})}\left(d_{\bar{z}}(u)\right) d o \rightarrow 0 \quad(s, t \nearrow \bar{t}) ;
$$

however, at a rate that may depend on $\bar{z}$.

Following Rauch [12] we now decompose $u=v+w$, where $v \in$ $C^{2}\left(\mathbb{R}^{3} \times[0, \infty[)\right.$ is the unique solution of the homogeneous wave equation

$$
v_{t t}-\Delta v=0
$$

with initial data (1.9) and

$$
w_{t t}-\Delta w+u^{5}=0, \quad w_{\mid t=0}=0=w_{t \mid t=0} .
$$

In particular, at $z_{0}=\left(x_{0}, t_{0}\right)$ we may express $w$ via (2.3) as follows

$$
w\left(z_{0}\right)=-\frac{1}{4 \pi} \int_{M_{0}\left(z_{0}\right)} \frac{u^{5}(x, t)}{t_{0}-t} d o(x, t) .
$$

Thus, and splitting integration over $M_{0}^{T}\left(z_{0}\right)$ and $M_{T}\left(z_{0}\right)$ for suitable $T$, we obtain

$$
\begin{aligned}
m_{0} & =\left|u\left(z_{0}\right)\right| \leq\left|v\left(z_{0}\right)\right|+\left|w\left(z_{0}\right)\right| \\
& \leq\left|v\left(z_{0}\right)\right|+\frac{m_{0}}{4 \pi} \int_{M_{T}\left(z_{0}\right)} \frac{u^{4}}{t_{0}-t} d o+\frac{1}{4 \pi} \int_{M_{0}^{T}\left(z_{0}\right)} \frac{|u|^{5}}{t_{0}-t} d o .
\end{aligned}
$$

By Hölder's inequality and Lemma 3.2, the last term

$$
\begin{aligned}
\int_{M_{0}^{T}\left(z_{0}\right)} \frac{|u|^{5}}{t_{0}-t} d o & \leq C\left(\left|t_{0}-T\right|^{-1 / 2}-\left|t_{0}\right|^{-1 / 2}\right)\left(\int_{M_{0}\left(z_{0}\right)}|u|^{6} d o\right)^{5 / 6} \\
& \leq C E_{0}^{5 / 6}\left|t_{0}-T\right|^{-1 / 2}
\end{aligned}
$$


Hence, if for some $T<t_{0}$ we have

$$
\int_{M_{T}\left(z_{0}\right)} \frac{u^{4}}{t_{0}-t} d o \leq 2 \pi
$$

from (3.2) and (3.3) we can bound

$$
m_{0} \leq 2\left|v\left(z_{0}\right)\right|+C E_{0}^{5 / 6}\left(\left|t_{0}-T\right|^{-1 / 2}-\left|t_{0}\right|^{-1 / 2}\right)
$$

in terms of the initial data, $z_{0}$, and $T$.

Now, by Hölder's inequality

$$
\int_{M_{T}\left(z_{0}\right)} \frac{u^{4}}{t_{0}-t} d o \leq\left(\int_{M_{T}\left(z_{0}\right)} \frac{|u|^{2}}{\left|t_{0}-t\right|^{2}} d o\right)^{1 / 2}\left(\int_{M_{T}\left(z_{0}\right)}|u|^{6} d o\right)^{1 / 2} .
$$

Let $\tilde{u}(y)=u\left(x_{0}+y, t_{0}-|y|\right)$. Then by Lemma 3.1 we have

$$
\begin{aligned}
\int_{M_{T}\left(z_{0}\right)} \frac{|u|^{2}}{\left|t_{0}-t\right|^{2}} d o & =\sqrt{2} \int_{B_{t_{0}-T}(0)} \frac{|\tilde{u}(y)|^{2}}{|y|^{2}} d y \\
& \leq C \int_{B_{t_{0}-T}(0)}|\nabla \tilde{u}|^{2} d y+C\left(\int_{B_{t_{0}-T}(0)}|\tilde{u}|^{6} d y\right)^{1 / 3} \\
& \leq C \int_{M_{T}\left(z_{0}\right)} d_{z_{0}}(u) d o+C\left(\int_{M_{T}\left(z_{0}\right)} d_{z_{0}}(u) d o\right)^{1 / 3} \\
& \leq C\left(E\left(u ; D\left(T ; z_{0}\right)\right)+E^{1 / 3}\left(u ; D\left(T ; z_{0}\right)\right)\right) .
\end{aligned}
$$

Thus

$$
\int_{M_{T}\left(z_{0}\right)} \frac{u^{4}}{t_{0}-t} d o \leq C\left(E\left(u ; D\left(T ; z_{0}\right)\right)+E^{2 / 3}\left(u ; D\left(T ; z_{0}\right)\right)\right) .
$$

With the special choice $T=0,(3.4-6)$ now lead immediately to Rauch's existence result:

Theorem 3.3. There exists a constant $\varepsilon_{0}>0$ such that (1.8), (1.9) for any $u_{0} \in C^{3}\left(\mathbb{R}^{3}\right), u_{1} \in C^{2}\left(\mathbb{R}^{3}\right)$ with energy

$$
E_{0}=\int_{\mathbb{R}^{3}}\left(\frac{\left|u_{1}\right|^{2}+\left|\nabla u_{0}\right|^{2}}{2}+\frac{\left|u_{0}\right|^{6}}{6}\right) d x<\varepsilon_{0}
$$

admits a global $C^{2}$-solution.

Remark 3.4. Estimates (3.2)-(3.6) also give the following local version of Rauch's theorem. Let $\bar{z}=(\bar{x}, \bar{t}), \bar{t}>0$ :

If $u \in C^{2}\left(\mathbb{R}^{3} \times[0, \bar{t})\right.$ is a solution to (1.8), (1.9), and if

$$
E(u ; D(T ; \bar{z}))<\varepsilon_{0}
$$

for some $T<\bar{t}$, then $u$ (and its first and second derivatives) can be uniformly a priori bounded on $K_{0}(\bar{z}) \backslash\{\bar{z}\}$ in terms of $T, \bar{z}$, and the initial data.

In fact, in this case $u$ can be extended as a solution of (1.8) to a full neighborhood of $\bar{z}$. Indeed, since $u \in C^{2}\left(\mathbb{R}^{3} \times[0, \bar{t})\right.$, condition (3.7) will be satisfied for all points $\tilde{z}=(\tilde{x}, \bar{t})$ with $\tilde{x}$ close to $\bar{x}$. 
Finally, observe that if $u$ (and hence its first and second derivatives) are uniformly bounded on $K_{0}(\bar{z}) \backslash\{\bar{z}\}$, condition (3.7) is automatically satisfied. Thus, in order to extend $u$ as a solution of (1.8) to a neighborhood of $\bar{z}$ it suffices to establish that

$$
\limsup _{\substack{z_{0} \in K(2)<\{\} \\ z_{0} \rightarrow 2}}\left|u\left(z_{0}\right)\right|<\infty .
$$

By steps (3.2)-(3.6) of the proof of Rauch's theorem then, in fact, it suffices to show that for some $T<\bar{t}$ there holds

$$
\limsup _{\substack{z_{0} \in K(2) \backslash\{2\} \\ z_{0} \rightarrow 2}} \int_{M_{T}\left(z_{0}\right)} \frac{u^{4}}{t_{0}-t} d o \leq 2 \pi .
$$

This will be important for our next topic.

\section{LARGE DATA}

We now show how the smallness assumption in Rauch's Theorem 3.3 can be removed. Again remark that it suffices to consider data $u_{0}, u_{1}$ with compact support.

Suppose by contradiction that $u$ does not extend globally. Then $u$ becomes unbounded in finite time $T$. Since the support of $u$ in $\mathbb{R}^{3} \times[0, T]$ is relatively compact there exists a "first" singular point $\bar{z}=(\bar{x}, \bar{t}), 0<\bar{t} \leq T$, such that

$$
|u(x, t)| \longrightarrow \infty
$$

for some sequence $x \rightarrow \bar{x}, t / \bar{t}$, and $\bar{t}$ is minimal with this property.

By Remark 3.4, in order to achieve a contradiction it suffices to establish condition (3.8).

Since $t=0$ in the following no longer plays a distinguished role, we may shift coordinates so that $\bar{z}=(0,0)$ and henceforth assume that $u$ is a $C^{2}$ solution of (1.8) on $\mathbb{R}^{3} \times\left[t_{1}, 0\right.$ [ for some $t_{1}<0$. As customary, the Landau symbol " $o(1)$ as $r \rightarrow 0$ " will denote error terms depending on a parameter $r$ that tend to 0 as $r \rightarrow 0$.

Observe that (1.8) and $E$ are invariant under scaling

$$
R \mapsto u_{R}(x, t)=R^{1 / 2} u(R x, R t),
$$

for any $R>0$.

Following Struwe [18, Lemma 2.3] we use the testing function $t u_{t}+x \cdot \nabla u+u$ to derive the following identity

$$
0=\left(u_{t t}-\Delta u+u^{5}\right)\left(t u_{t}+x \cdot \nabla u+u\right)=\frac{d}{d t}\left(t Q_{0}+u_{t} u\right)-\operatorname{div}\left(t P_{0}\right)+R_{0}
$$

where

$$
\begin{aligned}
& P_{0}=\frac{x}{t}\left(\frac{1}{2}\left|u_{t}\right|^{2}-\frac{1}{2}|\nabla u|^{2}-\frac{1}{6}|u|^{6}\right)+\left(u_{t}+\frac{x}{t} \cdot \nabla u+\frac{u}{t}\right) \nabla u, \\
& Q_{0}=\frac{1}{2}\left|u_{t}\right|^{2}+\frac{1}{2}|\nabla u|^{2}+\frac{1}{6}|u|^{6}+\left(\frac{x}{t} \cdot \nabla u\right) u_{t} \geq 0 \text { in } K_{t_{1}}, \\
& R_{0}=\frac{1}{3}|u|^{6} \geq 0 .
\end{aligned}
$$

Note that the multiplier $t u_{t}+x \cdot \nabla u+u$ is related to the generator of the scaled family $u_{R}$. As in Grillakis [6, (2.2)], we may rewrite (4.1) in the form

$$
0=t\left\{\frac{d}{d t}\left(Q_{0}+\frac{u_{t} u}{t}+\frac{u^{2}}{2 t^{2}}\right)-\operatorname{div} P_{0}\right\}+\left\{Q_{0}+\frac{u^{2}}{t^{2}}+R_{0}\right\} \text {. }
$$


If we integrate (4.1) over a truncated cone $K_{t}^{s}$, integrals involving $u_{t} u$ will appear. Using the function $\left(t^{2}+|x|^{2}\right) u_{t}+2 t x \cdot \nabla u+2 t u$ as a further multiplier, Grillakis succeeds in showing that

$$
\frac{1}{|t|} \int_{D(t)} u_{t} u d x \leq o(1) \rightarrow 0 \quad(t \nearrow 0) .
$$

With little more extra work this additional multiplier can be avoided.

As a first step, we obtain

Lemma 4.1 [18, Lemma 3.2]. There exists a sequence of numbers $t_{\ell} \nearrow 0$ such that

$$
\frac{1}{\left|t_{l}\right|} \int_{D\left(t_{l}\right)} u_{t} u d x \leq o(1)
$$

where $o(1) \rightarrow 0$ as $l \rightarrow \infty$.

For completeness we present the proof.

Proof. Consider $u_{l}(x, t)=2^{-l / 2} u\left(2^{-l} x, 2^{-l} t\right), l \in \mathbb{N}$, satisfying (1.8) with

$$
E\left(u_{l} ; D(t)\right)=E\left(u ; D\left(2^{-l} t\right)\right) \leq E_{0} ;
$$

moreover, (3.1) translates into the condition $\left(d(u):=d_{0}(u)\right)$

$$
\int_{M_{t_{1}}} d\left(u_{l}\right) d o \rightarrow 0
$$

as $l \rightarrow \infty$. First, suppose that

$$
\int_{D\left(t_{1}\right)} u_{l}^{2} d x \rightarrow 0 \quad(l \rightarrow \infty) .
$$

Then let $t_{l}=2^{-\ell} t_{1}$ and estimate

$$
\begin{aligned}
\frac{1}{\left|t_{l}\right|} \int_{D\left(t_{l}\right)} u_{t} u d x & \leq\left(\int_{D\left(t_{l}\right)}\left|u_{t}\right|^{2} d x\right)^{1 / 2}\left(\frac{1}{\left|t_{l}\right|^{2}} \int_{D\left(t a_{l}\right)} u^{2} d x\right)^{1 / 2} \\
& \leq\left(2 E\left(u ; D\left(t_{l}\right)\right)\right)^{1 / 2}\left(\frac{1}{\left|t_{1}\right|^{2}} \int_{D\left(t_{1}\right)} u_{l}^{2} d x\right)^{1 / 2} \rightarrow 0 \quad(l \rightarrow \infty)
\end{aligned}
$$

to achieve the claim.

Otherwise, there exist $C_{1}>0$ and a sequence $\Lambda$ of numbers $l \rightarrow \infty$ such that

$$
\liminf _{l \rightarrow \infty, l \in \Lambda} \int_{D\left(t_{1}\right)} u_{l}^{2} d x \geq C_{1} .
$$

For any $s \in\left[t_{1}, 0[\right.$, by Hölder's inequality

$$
\int_{D(s)} u_{l}^{2} d x \leq\left(\frac{4 \pi}{3}|s|^{3}\right)^{2 / 3}\left(\int_{D(s)}\left|u_{l}\right|^{6} d x\right)^{1 / 3} \leq C E_{0}^{1 / 3} s^{2} .
$$


Choose $s=s_{1}<0$ such that the latter is $\leq C_{1}$. Then by (4.3) we have

$$
\begin{aligned}
2 \int_{K_{t_{1}}^{s_{1}}}\left(u_{l}\right)_{t} u_{l} d z & =\int_{K_{t_{1}}^{s_{1}}} \frac{d}{d t}\left|u_{l}\right|^{2} d x \\
& =\int_{D\left(s_{1}\right)}\left|u_{l}\right|^{2} d x-\int_{D\left(t_{1}\right)}\left|u_{l}\right|^{2} d x+\frac{1}{\sqrt{2}} \int_{M_{t_{1}}^{s_{1}}}\left|u_{l}\right|^{2} d o \\
& \leq o(1) \rightarrow 0 \quad(l \rightarrow \infty, \quad l \in \Lambda) .
\end{aligned}
$$

We conclude that for suitable numbers $s_{l} \in\left[t_{1}, s_{1}\right], t_{l}=2^{-\ell} s_{l}, l \in \Lambda$, we have

$$
\frac{2}{\left|t_{l}\right|} \int_{D\left(t_{l}\right)} u_{t} u d x=\frac{2}{\left|s_{\ell}\right|} \int_{D\left(s_{l}\right)}\left(u_{l}\right)_{t} u_{l} d x \leq o(1) \rightarrow 0 \quad(l \rightarrow \infty, l \in \Lambda) .
$$

Relabelling, we obtain a sequence $\left(t_{l}\right)_{l \in \mathbb{N}}$, as desired.

Lemma 4.2 [18, Lemma 2.2]. For any $l \in \mathbb{N}$ there holds

$$
\begin{aligned}
& \frac{1}{3\left|t_{l}\right|} \int_{K_{t_{l}}}|u|^{6} d z+\int_{D\left(t_{l}\right)}\left(\frac{1}{2}\left|u_{t}\right|^{2}+\frac{1}{2}|\nabla u|^{2}+u_{t}\left(\frac{x}{t} \cdot \nabla u\right)+\frac{1}{6}|u|^{6}\right) d x \\
& \quad \leq o(1) \rightarrow 0 \quad(l \rightarrow \infty) .
\end{aligned}
$$

Again we give the proof for completeness.

Proof. For $s \in\left[t_{l}, 0\left[\right.\right.$ integrate (4.1) over $K_{t_{l}}^{s}$ to obtain

$$
\begin{aligned}
0= & \int_{D(s)}\left(s Q_{0}+u_{t} u\right) d x+\frac{1}{\sqrt{2}} \int_{M_{t_{l}}^{s}}\left(t Q_{0}+u_{t} u-x \cdot P_{0}\right) d o \\
& +\left|t_{l}\right| \int_{D\left(t_{l}\right)} Q_{0} d x+\int_{K_{t_{l}}^{s}} R_{0} d x-\int_{D\left(t_{l}\right)} u_{t} u d x .
\end{aligned}
$$

Now, $Q_{0}$ is dominated by the energy density $e(u)$. Thus, and using Hölder's inequality as in (4.4), (4.5), the first term is of order $|s|$ and hence vanishes as $s \nearrow 0$. Moreover, on $M_{t_{l}}$ we have

$$
\begin{aligned}
\mid t Q_{0} & +u_{t} u-x \cdot P_{0} \mid \\
& =\left.|t||| \nabla u\right|^{2}-\left|\frac{x}{|x|} \cdot \nabla u\right|^{2}+\frac{1}{3}|u|^{6}-\frac{u\left(t u_{t}+x \cdot \nabla u\right)}{t^{2}} \mid \\
& \leq\left|t_{l}\right|\left(3 d_{0}(u)+\frac{|u|^{2}}{t^{2}}\right) .
\end{aligned}
$$

Hence by (3.1) and Hardy's inequality Lemma 3.1 the second term is of order $o(1)\left|t_{l}\right|$, where $o(1) \rightarrow 0$ as $l \rightarrow \infty$. Thus, by Lemma 4.1 we have

$\frac{1}{\left|t_{l}\right|} \int_{K_{\ell_{l}}^{s}} R_{0} d z+\int_{D\left(t_{l}\right)} Q_{0} d x \leq \frac{1}{\left|t_{l}\right|} \int_{D\left(t_{l}\right)} u_{t} u d x+o(1) \leq o(1) \rightarrow 0 \quad(l \rightarrow \infty)$, which is the desired conclusion.

Now we use (4.1) in its equivalent form (4.2) to derive a stronger version of Lemma 4.1. 
Lemma 4.3. There exists a sequence of numbers $\bar{t}_{l} \nearrow 0$ such that the conclusion of Lemma 4.1 holds for $\left(\bar{t}_{l}\right)$ while in addition we have

$$
2 \leq \bar{t}_{l} / \bar{t}_{l+1} \leq 4
$$

for all $l \in \mathbb{N}$.

Proof. First observe that by Hölder's inequality and Lemma 4.2 for any $m \in \mathbb{N}$ we have

$\int_{D\left(t_{m}\right)} \frac{|u|^{2}}{|t|^{2}} d x \leq C\left(\int_{D\left(t_{m}\right)}|u|^{6} d x\right)^{1 / 3} \leq C\left(\int_{D\left(t_{m}\right)} Q_{0} d x\right)^{1 / 3} \rightarrow 0 \quad(m \rightarrow \infty)$, where $\left(t_{m}\right)$ is determined in Lemma 4.1. Divide (4.2) by $t$ and integrate over the cone $K_{t_{l}}^{t_{m}}$ for $m \geq l$ to obtain

$$
\begin{aligned}
\int_{D\left(t_{l}\right)} & \left(Q_{0}+\frac{u_{t} u}{t}+\frac{u^{2}}{2 t^{2}}\right) d x+\int_{K_{t_{t}}^{t_{m}}}\left(\frac{Q_{0}}{|t|}+\frac{|u|^{2}}{|t|^{3}}+\frac{R_{0}}{|t|}\right) d z \\
= & \int_{D\left(t_{m}\right)}\left(Q_{0}+\frac{u_{t} u}{t}+\frac{u^{2}}{2 t^{2}}\right) d x+\int_{M_{t_{l}}^{t_{m}}}\left(Q_{0}+\frac{u_{t} u}{t}+\frac{u^{2}}{2 t^{2}}-\frac{x}{t} \cdot P_{0}\right) d o .
\end{aligned}
$$

By the preceding remark the first term on the right vanishes as we let $m \rightarrow \infty$, while by (3.1) the integral over $M_{t_{l}}^{t_{m}}$ becomes arbitrarily small as $m \geq l \rightarrow \infty$. Finally, by Lemma 4.1 , we have

$$
\int_{D\left(t_{l}\right)} \frac{u_{t} u}{t} d x=-\frac{1}{\left|t_{l}\right|} \int u_{t} u d x \geq o(1) \rightarrow 0 \quad(l \rightarrow \infty)
$$

All remaining terms being nonnegative, we thus obtain the estimate

$$
\int_{K_{t_{l}}} \frac{|u|^{2}}{|t|^{3}} d z=\int_{t_{l}}^{0}\left(\frac{1}{|t|} \int_{D(t)} \frac{|u|^{2}}{|t|^{2}} d x\right) d t \leq o(1) \rightarrow 0 \quad(l \rightarrow \infty) .
$$

Hence for any $\bar{t} \in\left[t_{l} / 2,0[\right.$ there also holds

$$
o(1) \geq \frac{1}{\bar{t}} \int_{2 \bar{t}}^{\bar{t}}\left(\int_{D(t)} \frac{|u|^{2}}{|t|^{2}} d x\right) d t \geq \inf _{2 \bar{t} \leq t \leq \bar{t}} \int_{D(t)} \frac{|u|^{2}}{|t|^{2}} d t,
$$

where $o(1) \rightarrow 0$ if $l \rightarrow \infty$.

To construct the sequence $\left(\bar{t}_{l}\right)$, now choose $\bar{t}_{1}=t_{1}$ and proceed by induction. Suppose $\bar{t}_{l}, l=1, \ldots, L$, have been defined already. Let $\bar{t}_{L+1} \in\left[\frac{\bar{t}_{L}}{2}, \frac{\bar{t}_{L}}{4}[\right.$ be chosen such that

$$
\int_{D\left(i_{L+1}\right)} \frac{|u|^{2}}{|t|^{2}} d x \leq 2 \inf _{\frac{i_{t}}{2} \leq t \leq \frac{i_{L}}{4}} \int_{D(t)} \frac{|u|^{2}}{|t|^{2}} d x .
$$

Clearly, this procedure yields a sequence $\left(\bar{t}_{l}\right)$ such that $2 \leq \bar{t}_{l} / \bar{t}_{l+1} \leq 4$ for all $l$ and

By (4.4) then

$$
\int_{D\left(\bar{t}_{l}\right)} \frac{|u|^{2}}{|t|^{2}} d x \rightarrow 0 \quad(l \rightarrow \infty)
$$

$$
\frac{1}{\left|\bar{t}_{l}\right|} \int_{D\left(\bar{t}_{\ell}\right)} u_{t} u d x \rightarrow 0 \quad(l \rightarrow \infty),
$$

concluding the proof.

To simplify notation, we will assume that $t_{l}=\bar{t}_{l}$ for all $l$, initially. 
The radial case. At this point we can indicate how the decay estimate Lemma 4.2 and Lemma 4.3 imply regularity of solutions in the radial case. First observe that for radially symmetric data $u_{0}(x)=u_{0}(|x|)$, etc., the unique local $C^{2}$-solution $u$ to (1.8), (1.9) again is radially symmetric, that is, $u(x, t)=$ $u(|x|, t)$.

Note that this implies that blow-up can only occur on the line $x=0$. Indeed, if $u$ is regular on $K_{0}(\bar{z}) \backslash\{\bar{z}\}$ and blows up at $\bar{z}=(\bar{z}, \bar{t})$, the same will be true for any point $z=(x, \bar{t})$ with $|x|=|\bar{x}|=\bar{r}$. Now, if $\bar{x} \neq 0$, given any $K \in \mathbb{N}$ we can choose points $x_{k} \in \mathbb{R}^{3}$ with $\left|x_{k}\right|=\bar{r}, 1 \leq k \leq K$, and $T \in[0, \bar{t}[$ such that

$$
D\left(T ; z_{k}\right) \cap D\left(T ; z_{l}\right)=\varnothing
$$

for all $k \neq l$, where $z_{k}=\left(x_{k}, T\right), k=1, \ldots, K$. Moreover, by Remark 3.4

$$
E\left(u ; D\left(T ; z_{k}\right)\right) \geq \varepsilon_{0}>0
$$

for all $k$, while by Lemma 3.2

$$
\begin{aligned}
K \varepsilon_{0} & \leq \sum_{k=1}^{K} E\left(u ; D\left(T ; z_{k}\right)\right)=E\left(u ; \bigcup_{k=1}^{K} D\left(T ; z_{k}\right)\right) \leq E(u ; D(T ;(0, \bar{r}+\bar{t}))) \\
& \leq E(u ; D(0 ;(0, \bar{r}+\bar{t}))) \leq E_{0}
\end{aligned}
$$

independently of $K$. Thus, blow-up may first occur on the line $x=0$, only. (See Figure 1.)

Let blow-up occur at $(0, \bar{t})$. Shifting time by $\bar{t}$ then we may assume that $u(x, t)=u(|x|, t)$ is regular on $\mathbb{R}^{3} \times\left[t_{1}, 0[\right.$ and blows up at the origin. As a second step we estimate the speed of blow-up. Again observe that (1.8) is invariant under scaling

$$
u \longmapsto u_{R}(x, t)=R^{1 / 2} u(R x, R t) .
$$

This suggests that $u(z) \sim|z|^{-1 / 2}$. In fact, the following result holds.

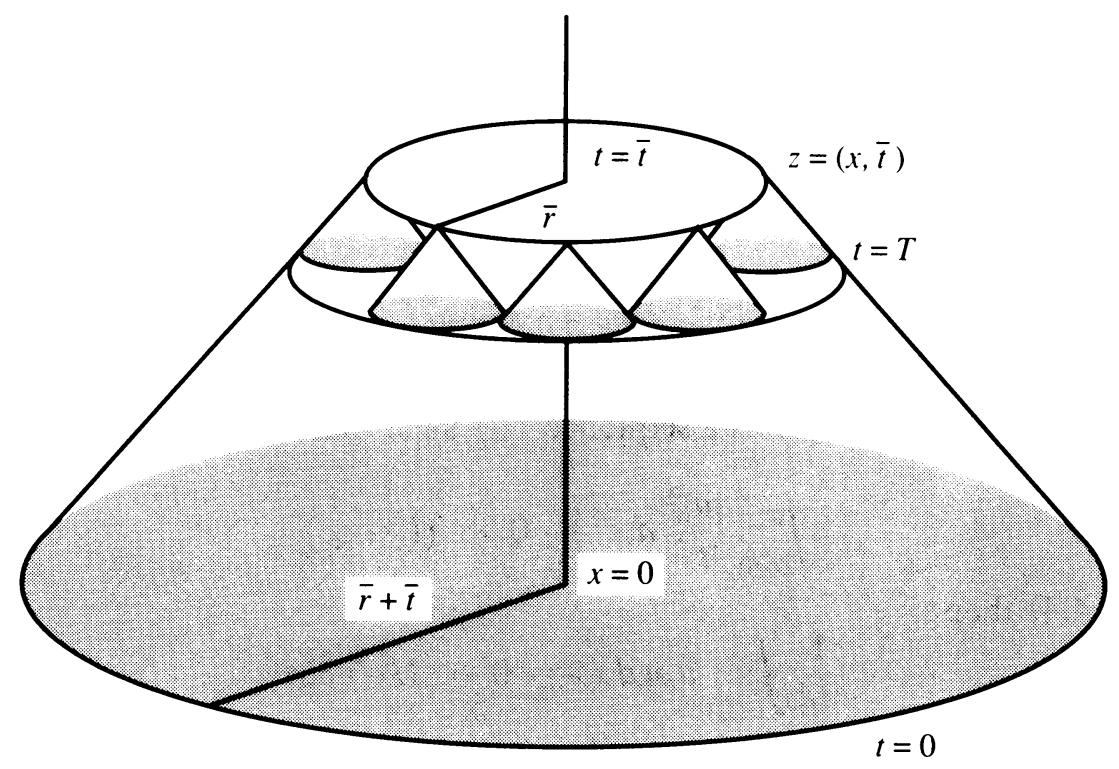

FIGURE 1 . The energy at the basis of each cone is $\geq \varepsilon_{0}$. 


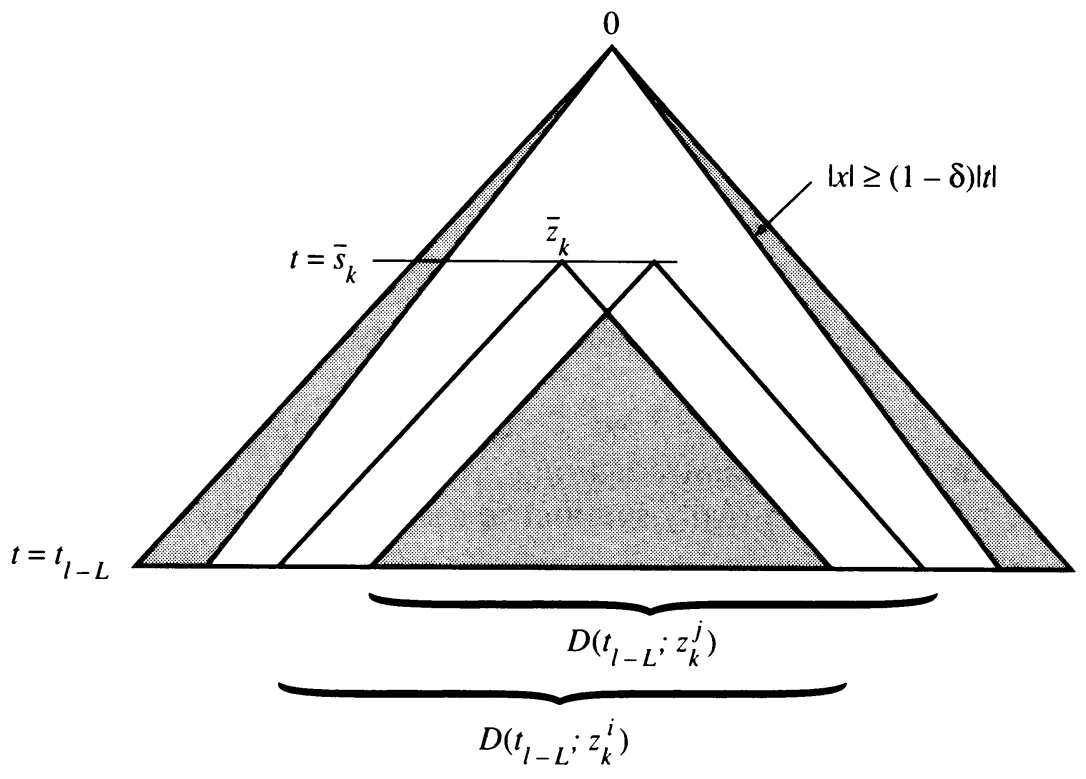

FIGURE 2. Overlap of the cones $K_{t_{l-L}}\left(z_{k}^{j}\right)$.

Lemma 4.4 [18; Lemma 3.3].

$$
4 \varepsilon_{1}:=\limsup _{\substack{z=(x, k) \rightarrow 0 \\ z \in K_{t_{1}}}}\left(|u(z)| \cdot|z|^{1 / 2}\right)>0 .
$$

The proof of Lemma 4.4 is rather involved and will not be presented here.

We can now conclude the regularity proof. Let $\bar{z}_{k}=\left(\bar{x}_{k}, \bar{s}_{k}\right) \in K_{t_{1}}$ satisfy

$$
\left|u\left(\bar{z}_{k}\right)\right|=\sup _{z \in K_{t_{1}}\left(\bar{z}_{k}\right)}|u(z)| \geq 2 \varepsilon_{1}\left|\bar{z}_{k}\right|^{-\frac{1}{2}} .
$$

Choose a sequence $l=l(k) \rightarrow \infty(k \rightarrow \infty)$ such that

$$
t_{l+1} \geq \bar{s}_{k} \geq t_{l}
$$

with $\left(t_{l}\right)$ as in Lemma 4.3. By (3.2)-(3.3) we may fix $L \in \mathbb{N}$ independent of $k$ such that for large $k$ there holds

$$
\begin{aligned}
\left|u\left(\bar{z}_{k}\right)\right|\left(1-\frac{1}{4 \pi} \int_{M_{t_{l-L}}\left(\bar{z}_{k}\right)} \frac{u^{4}}{\bar{s}_{k}-t} d o\right) & \leq C+C\left(\left|\bar{s}_{k}-t_{l-L}\right|^{-1 / 2}-\left|\bar{s}_{k}-t_{1}\right|^{-1 / 2}\right) E_{0}^{5 / 6} \\
& \leq C+C 2^{-L / 2}\left|\bar{s}_{k}\right|^{-1 / 2} E_{0}^{5 / 6} \leq \varepsilon_{1}\left|\bar{z}_{k}\right|^{-1 / 2}
\end{aligned}
$$

where $E_{0}=E\left(u ; D\left(t_{1}, 0\right)\right)$ is the initial energy.

Thus, by choice of $\bar{z}_{k}$ and (3.6) we conclude that

$$
E\left(u ; D\left(t_{l-L} ; \bar{z}_{k}\right)\right) \geq \varepsilon_{0} \text { for all } k .
$$

Given $J \in \mathbb{N}$, for each $k \in \mathbb{N}$ choose $J$ points $x_{k}^{j}, j=1, \ldots, J$, equidistributed on the sphere $\left|x_{k}^{j}\right|=\left|\bar{x}_{k}\right|$. Let $z_{k}^{j}=\left(x_{k}^{j}, \bar{s}_{k}\right)$. Note that there exists $\delta=\delta(J, L)>0$ such that $\left(x, t_{l-L}\right) \in D\left(t_{l-L} ; z_{k}^{i}\right) \cap D\left(t_{l-L} ; z_{k}^{j}\right), i \neq j$, implies that $|x| \leq(1-\delta) t_{l-L}$. (See Figure 2.) 
Hence by Lemma 4.2 we have

$$
\begin{aligned}
J \varepsilon_{0} & \leq \sum_{j=1}^{J} E\left(u ; D\left(t_{l-L} ; z_{k}^{j}\right)\right) \\
& \leq E\left(u ; D\left(t_{l-L} ; 0\right)\right)+\sum_{i \neq j} E\left(u ; D\left(t_{l-L} ; z_{k}^{i}\right) \cap D\left(t_{l-L} ; z_{k}^{j}\right)\right) \\
& \leq E_{0}+C(J, \delta) \int_{D\left(t_{l-L}\right)} Q_{0} d x \leq E_{0}+o(1),
\end{aligned}
$$

where $o(1) \rightarrow 0$ as $k \rightarrow \infty$ for any fixed $J$. Choosing $J$ large, for sufficiently large $k \in \mathbb{N}$ we thus obtain a contradiction. Hence, $u$ is uniformly bounded on $K_{t_{1}}$ and the proof in the radially symmetric case is complete.

General data. Finally, we present Grillakis' work on the general case. The key observation is that the decay Lemma 4.2 suffices to establish (3.8), directly. However, this is not at all easy to see.

Fix $z_{0}=\left(x_{0}, t_{0}\right) \in K \backslash\{0\}$ arbitrarily. Denote $y=x-x_{0}, \hat{y}=\frac{y}{|y|}, \hat{x}=\frac{x}{|x|}$. Divide (4.2) by $t$ and for $s>t_{0}$ integrate over $K_{t_{l}}^{s} \backslash K\left(z_{0}\right)$ to obtain the relation

$$
\begin{aligned}
0= & \int_{D(s)}\left(Q_{0}+\frac{u_{t} u}{t}+\frac{u^{2}}{2 t^{2}}\right) d x-\int_{D\left(t_{t}\right) \backslash D\left(t_{l} ; z_{0}\right)}\left(Q_{0}+\frac{u_{t} u}{t}+\frac{u^{2}}{2 t^{2}}\right) d x \\
& +\frac{1}{\sqrt{2}} \int_{M_{t_{l}}^{s}}\left(Q_{0}+\frac{u_{t} u}{t}+\frac{u^{2}}{2 t^{2}}-\hat{x} \cdot P\right) d o \\
& -\frac{1}{\sqrt{2}} \int_{M_{t_{l}}\left(z_{0}\right)}\left(Q_{0}+\frac{u_{t} u}{t}+\frac{u^{2}}{2 t^{2}}-\hat{y} \cdot P\right) d o \\
& +\int_{K_{t_{l}}^{s} \backslash K\left(z_{0}\right)}\left(\frac{\left.R_{0}+Q_{0}+\frac{u^{2}}{2 t^{2}}\right) d z=I+\cdots+V .}{t}\right)
\end{aligned}
$$

(See Figure 3.)

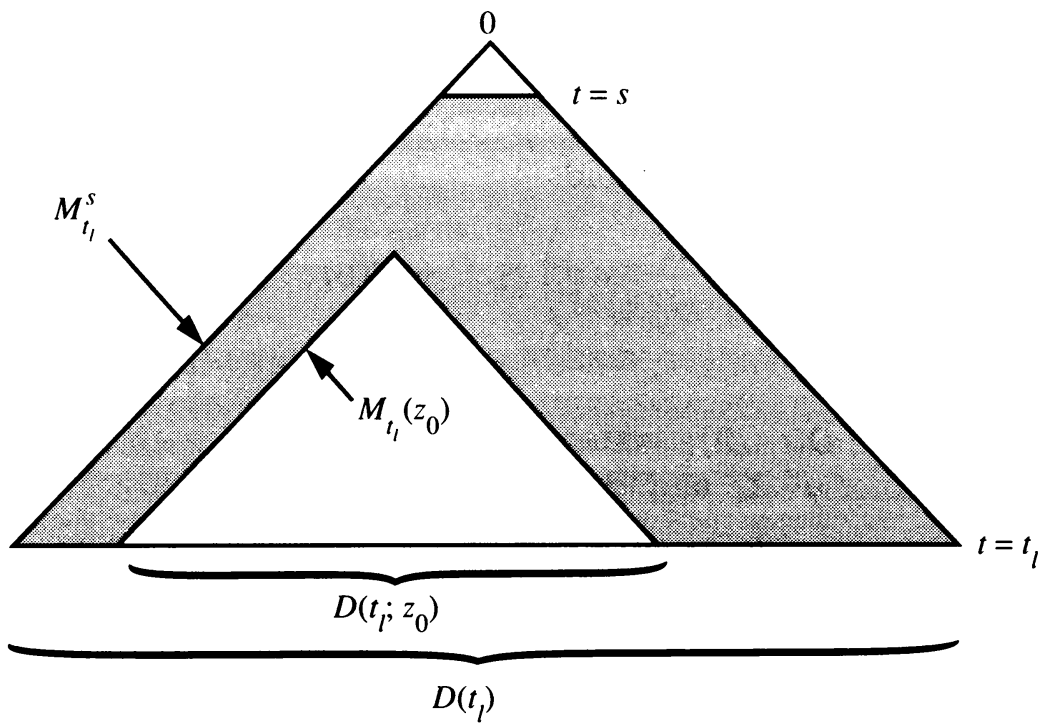

FIGURE 3 
By Hölder's inequality (4.4), (4.5) and Lemma 4.2 the first term I $\rightarrow 0$ if we choose $s=t_{k}$ with $k \rightarrow \infty$. Similarly, II $\rightarrow 0$ if $l \rightarrow \infty$. By (3.1) also III $\rightarrow 0$ as $l \rightarrow \infty$. Finally $\mathrm{V} \leq 0$. Thus we obtain the estimate for any $z_{0} \in K \backslash\{0\}$,

$$
\int_{M_{t_{l}\left(z_{0}\right)}}\left(Q_{0}+\frac{u_{t} u}{t}+\frac{u^{2}}{2 t^{2}}-\hat{y} \cdot P\right) d o \leq o(1) \rightarrow 0 \quad(l \rightarrow \infty),
$$

with error term $o(1)$ independent of $z_{0}$.

By a beautiful geometric reasoning, Grillakis [6] now proceeds to bound (3.8) in terms of (4.6) of (4.6). Let $r=|x|$; then we may rewrite

$$
\begin{aligned}
A:= & Q_{0}+\frac{u_{t} u}{t}+\frac{u^{2}}{2 t^{2}}-\hat{y} \cdot P \\
= & \left(1-\hat{x} \cdot \hat{y} \frac{r}{t}\right) \frac{1}{2}\left|u_{t}\right|^{2}+\left(1+\hat{x} \cdot \hat{y} \frac{r}{t}\right)\left(\frac{1}{2}|\nabla u|^{2}+\frac{1}{6}|u|^{6}\right) \\
& +\frac{1}{t}\left(u_{t}-\hat{y} \cdot \nabla u\right) u+\frac{r}{t} u_{t} \hat{x} \cdot \nabla u-u_{t} \hat{y} \cdot \nabla u-\frac{r}{t}(\hat{x} \cdot \nabla u)(\hat{y} \cdot \nabla u)+\frac{u^{2}}{2 t^{2}} .
\end{aligned}
$$

Introducing $u_{\sigma}=\hat{y} \cdot \nabla u, \alpha=\hat{x}-\hat{y}(\hat{y} \cdot \hat{x}),|\alpha| u_{\alpha}=\alpha \cdot \nabla u, \Omega u=\nabla u-\hat{y} u_{\sigma}$, this expression becomes

$$
\begin{aligned}
= & \left(1-\hat{x} \cdot \hat{y} \frac{r}{t}\right) \frac{1}{2}\left(u_{t}-u_{\sigma}\right)^{2}+\left(1+\hat{x} \cdot \hat{y} \frac{r}{t}\right)\left(\frac{1}{2}|\Omega u|^{2}+\frac{1}{6}|u|^{6}\right) \\
& +\frac{r}{t}|\alpha|\left(u_{t}-u_{\sigma}\right) u_{\alpha}+\frac{u}{t}\left(u_{t}-u_{\sigma}\right)+\frac{u^{2}}{2 t^{2}} .
\end{aligned}
$$

Now let $\hat{x} \cdot \hat{y}=\cos \delta,|\alpha|=\sin \delta$ and for brevity denote $\frac{1}{\sqrt{2}}\left(u_{t}-u_{\sigma}\right)=u_{\rho}$. (See Figure 4 on p. 78.) Then the above

$$
\begin{aligned}
A= & \left(1-\frac{r}{t} \cos \delta\right)\left|u_{\rho}\right|^{2}+\left(1+\frac{r}{t} \cos \delta\right)\left(\frac{1}{2}|\Omega u|^{2}+\frac{1}{6}|u|^{6}\right) \\
& +\sqrt{2} \frac{r}{t}|\sin \delta| u_{\rho} u_{\alpha}+\sqrt{2} \frac{u_{\rho} u}{t}+\frac{u^{2}}{2 t^{2}} \\
= & : A_{0}+\sqrt{2} \frac{u_{\rho} u}{t}+\frac{u^{2}}{2 t^{2}} .
\end{aligned}
$$

Note that if we estimate $\left|u_{\alpha}\right| \leq|\Omega u|$, in particular, we have

$$
\begin{aligned}
& A_{0} \geq\left(1-\frac{r}{t} \cos \delta\right)\left|u_{\rho}\right|^{2}+\left(1+\frac{r}{t} \cos \delta\right)\left(\frac{1}{2}\left|u_{\alpha}\right|^{2}+\frac{1}{6}|u|^{6}\right)+\sqrt{2} \frac{r}{t}|\sin \delta| u_{\rho} u_{\alpha} \\
& \quad \geq\left(1+\frac{r}{t}\right)\left(\left|u_{\rho}\right|^{2}+\left.\frac{1}{2} u_{\alpha}\right|^{2}+\frac{1}{6}|u|^{6}\right)-\frac{r}{2 t}\left(\sqrt{2} \sqrt{1+\cos \delta} u_{\rho}-\sqrt{1-\cos \delta} u_{\alpha}\right)^{2} \\
& \quad \geq 0
\end{aligned}
$$

Now for any $\varepsilon>0$ there is a constant $C=C(\varepsilon)$ such that for any $z_{0} \in K$ and any $z \in M^{C t_{0}}\left(z_{0}\right)$ we may estimate

$$
-\sqrt{2} \frac{r}{t}|\sin \delta| \leq \varepsilon, \quad-\frac{r}{t} \cos \delta \geq \frac{1}{2} .
$$

In fact, for $z=(x, t) \in M^{C t_{0}}\left(z_{0}\right)$ we have

$$
|| x|-| y|| \leq|y-x|=\left|x_{0}\right| \leq\left|t_{0}\right| \leq \frac{\left|t-t_{0}\right|}{C-1}=\frac{|y|}{C-1} .
$$




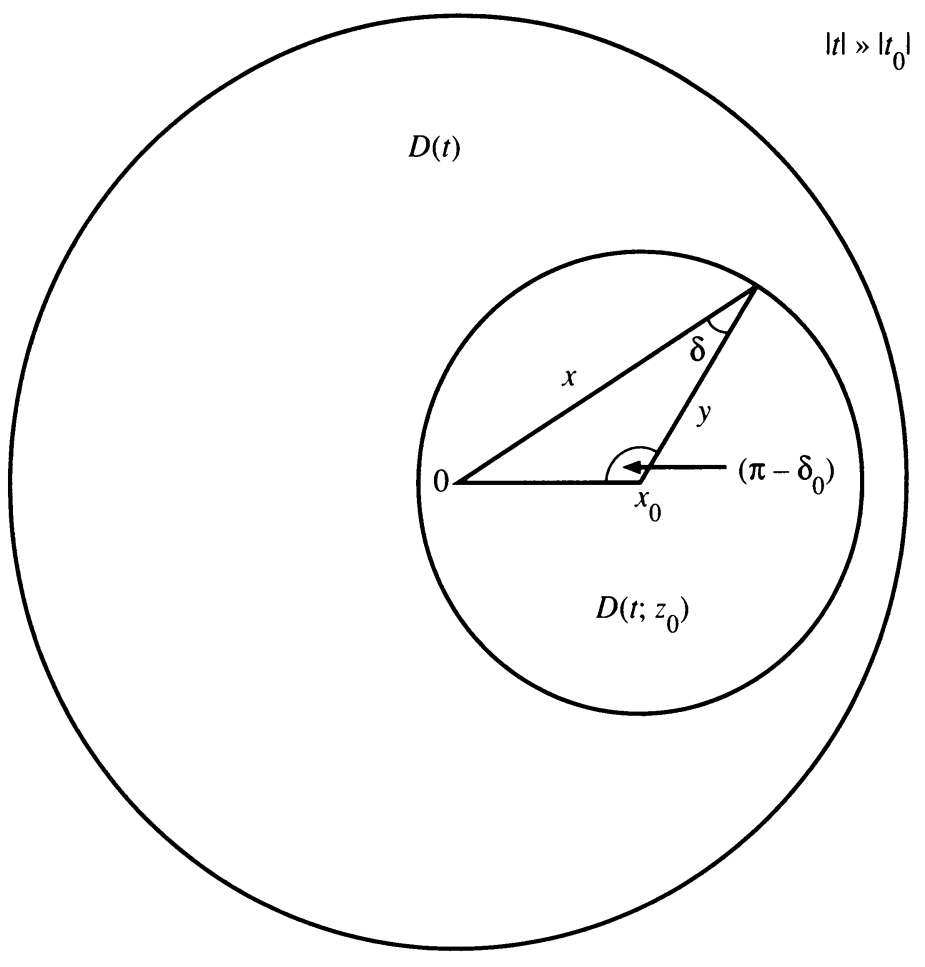

FIGURE 4

Hence

$$
\hat{x} \cdot \hat{y}=\cos \delta=\frac{x}{|x|} \cdot \frac{y}{|y|} \geq 1-\left|\frac{y}{|y|}-\frac{x}{|x|}\right| \geq 1-2 \frac{\left|x_{0}\right|}{|y|} \geq 1-\frac{2}{C-1}
$$

while

$$
1 \geq-\frac{r}{t}=\frac{|y|}{\left|t-t_{0}\right|} \cdot \frac{\left|t-t_{0}\right|}{|t|} \cdot \frac{|x|}{|y|} \geq\left(1-\frac{1}{C}\right)\left(1-\frac{1}{C-1}\right) .
$$

This yields the following estimate.

Lemma 4.5. For any $\varepsilon>0$, any $z_{0} \in K$, letting $C=C(\varepsilon)$ be determined as above, if $t_{k} \leq C t_{0}$ we have

Proof. Estimate

$$
\int_{M_{l}^{t_{k}}\left(z_{0}\right)} A d o \geq \frac{1}{2} \int_{M_{t_{l}}^{t_{k}}\left(z_{0}\right)}\left|u_{\rho}\right|^{2} d o-\varepsilon E_{0}
$$

$$
\left|\frac{\sqrt{2} u_{\rho} u}{t}\right| \leq\left|u_{\rho}\right|^{2}+\frac{u^{2}}{2 t^{2}}
$$

Hence by (4.7) and our choice of $C(\varepsilon)$, for $z \in M_{t_{l}}^{C t_{0}}\left(z_{0}\right)$ we have

$$
A \geq \frac{1}{2}\left|u_{\rho}\right|^{2}-\varepsilon\left|u_{\rho} u_{\alpha}\right| \geq \frac{1}{2}\left|u_{\rho}\right|^{2}-\frac{1}{\sqrt{2}} \in d_{z_{0}}(u),
$$

which in view of Lemma 3.2 proves the claim. 
Observe that $u_{\rho}$ may be interpreted as a tangent derivative along $M\left(z_{0}\right)$. In fact, let $\Phi$ be the map

$$
\Phi: y \mapsto\left(x_{0}+y, t_{0}-|y|\right)
$$

and let

$$
v(y)=u(\Phi(y))
$$

whenever the latter is defined. Then the radial derivative $v_{s}$ of $v$ is given by

$$
v_{s}=\frac{y \cdot \nabla v}{|y|}=u_{\sigma}-u_{t}=-\sqrt{2} u_{\rho} .
$$

Lemma 4.6 (Grillakis [6, (2.23)]). For any $z_{0} \in K$ and any $C \geq 0$ there holds

$$
\int_{M_{(1+C) t_{0}}\left(z_{0}\right)} \frac{u_{\rho} u}{t} d o \geq(1+\ln (1+C)) \cdot o(1)
$$

where $o(1) \rightarrow 0$ if $(1+C) t_{0} \geq t_{l}$ and $l \rightarrow \infty$.

Proof. Introducing $y$ as new variable via (4.9), (4.10), we have

$$
\int_{M_{(1+C) t_{0}\left(z_{0}\right)}} \frac{u_{\rho} u}{t} d o=\int_{B_{C\left|t_{0}\right|}} v_{s} \frac{v}{|y|-t_{0}} d y=\int_{S_{1}}\left(\int_{0}^{C\left|t_{0}\right|} v_{s} \frac{v}{s-t_{0}} s^{2} d s\right) d o .
$$

Upon integrating by parts, this gives

$$
\begin{aligned}
& \int_{S_{1}}\left(\int_{0}^{C\left|t_{0}\right|} \frac{\partial_{s}\left(v^{2} / 2\right)}{s-t_{0}} s^{2} d s\right) d o \\
& \quad=\int_{S_{1}} \int_{0}^{C\left|t_{0}\right|}\left(-\frac{v^{2} s}{s-t_{0}}+\frac{v^{2} s^{2}}{2\left(s-t_{0}\right)^{2}}\right) d s d o+\frac{1}{2(1+C)\left|t_{0}\right|} \int_{S_{C\left|t_{0}\right|}} v^{2} d o \\
& \quad \geq-\int_{B_{C\left|t_{0}\right|}} \frac{v^{2}}{|y|\left(|y|-t_{0}\right)} d y=-\frac{1}{\sqrt{2}} \int_{M_{(1+C) t_{0}}\left(z_{0}\right)} \frac{u^{2}}{t\left(t-t_{0}\right)} d o(x, t) \\
& \quad=-\int_{(1+C) t_{0}}^{t_{0}} \frac{1}{|t|}\left(\frac{1}{\left|t-t_{0}\right|} \int_{\partial D\left(t ; z_{0}\right)} u^{2} d o(x)\right) d t .
\end{aligned}
$$

Now by Hardy's inequality Lemma 3.1.(iii) we have

$$
\begin{aligned}
& \left(\frac{1}{\left|t-t_{0}\right|} \int_{\partial D\left(t ; z_{0}\right)} u^{2} d o\right)^{2} \leq C \int_{\partial D\left(t ; z_{0}\right)} u^{4} d o \\
& \quad \leq C\left\{\left(\int_{D\left(t ; z_{0}\right)}|\nabla u|^{2} d x\right)^{1 / 2}+\left(\int_{D\left(t ; z_{0}\right)}|u|^{6} d x\right)^{1 / 6}\right\} \cdot\left(\int_{D\left(t ; z_{0}\right)}|u|^{6} d x\right)^{1 / 2} \\
& \quad \leq C\left(E_{0}\right)\left(\int_{D\left(t ; z_{0}\right)}|u|^{6} d x\right)^{1 / 2} .
\end{aligned}
$$

Hence

$$
\int_{M_{(1+C) t_{0}\left(z_{0}\right)}} \frac{u_{\rho} u}{t} d o \geq-C \int_{(1+C) t_{0}}^{t_{0}} \frac{1}{|t|}\left(\int_{D(t)}|u|^{6} d x\right)^{1 / 4} d t
$$


with $C$ depending on $E_{0}$ only. By Lemma 4.2 the latter can be controlled as follows. Let $k, K \in \mathbb{N}$ be determined such that

$$
t_{k} \leq(1+C) t_{0}<t_{k+1} \leq t_{K} \leq t_{0}<t_{K+1} .
$$

Note that by Lemma 4.3

$$
1+C \geq \frac{t_{k+1}}{t_{K}} \geq 2^{K-(k+1)},
$$

whence

$$
K-k \leq 1+\log _{2}(1+C) .
$$

Estimate

$$
\int_{(1+C) t_{0}}^{t_{0}} \frac{1}{|t|}\left(\int_{D(t)}|u|^{6} d x\right)^{1 / 4} d t \leq \sum_{i=k}^{K} \int_{t_{i}}^{t_{i+1}} \frac{1}{|t|}\left(\int_{D(t)}|u|^{6} d x\right)^{1 / 4} d t
$$

By Hölder's inequality, this is

$$
\leq C \sum_{i=k}^{K} \frac{\left|t_{i}-t_{i+1}\right|^{3 / 4}}{\left|t_{i+1}\right|}\left(\int_{K_{t_{i}}^{t_{i+1}}}|u|^{6} d z\right)^{1 / 4}
$$

and by Lemma 4.3

$$
\leq C \sum_{i=k}^{K}\left(\frac{1}{\left|t_{i}\right|} \int_{K_{t_{i}}}|u|^{6} d z\right)^{1 / 4} .
$$

Finally, use Lemma 4.2 to see that this is

$$
\leq(K-k+1) o(1) \leqq(1+\ln (1+C)) o(1)
$$

where $o(1) \rightarrow$ if $(1+C) t_{0} \geq t_{l}$ and $l \rightarrow \infty$.

Combining Lemmas 4.5 and 4.6 it follows that for any $\varepsilon>0$, if we choose $t_{k} \leq C(\varepsilon) t_{0}<t_{k+1}$, we can estimate

$$
\begin{aligned}
o(1) & \geq \int_{M_{t_{l}}\left(z_{0}\right)} A d o \\
& \geq \frac{1}{2} \int_{M_{t_{l}}^{t_{k}}\left(z_{0}\right)}\left|u_{\rho}\right|^{2} d o-\varepsilon E_{0}+\int_{M_{t_{k}}\left(z_{0}\right)} A_{0} d o-o(1)(1+\ln (1+C(\varepsilon))),
\end{aligned}
$$

where $o(1) \rightarrow 0$ as $l \rightarrow \infty$. To estimate $A_{0}$ on $M_{t_{k}}\left(z_{0}\right)$ now introduce the new angle $\delta_{0}$, where $\left|x_{0}\right|=r_{0}, \hat{x}_{0}=\frac{x_{0}}{r_{0}}, \hat{x}_{0} \cdot \hat{y}=\cos \delta_{0}$. (See Figure 5.) Again $y=x-x_{0}$, and $|y|=\sigma=t_{0}-t$. With this notation

$$
\begin{gathered}
r \cos \delta=\hat{x} \cdot \hat{y} r=x \cdot \hat{y}=y \cdot \hat{y}+x_{0} \cdot \hat{y}=\sigma+r_{0} \cos \delta_{0}, \\
|\sin \delta|=|\alpha|=\left|\frac{x-(x \cdot \hat{y}) \hat{y}}{r}\right|=\left|\frac{x_{0}-\left(x_{0} \cdot \hat{y}\right) \hat{y}}{r}\right|=\frac{r_{0}}{r}\left|\sin \delta_{0}\right| .
\end{gathered}
$$

Hence, by (4.7),

$$
\begin{aligned}
A_{0}= & \left(1-\frac{\sigma}{t}-\frac{r_{0}}{t} \cos \delta_{0}\right)\left|u_{\rho}\right|^{2}+\left(1+\frac{\sigma}{t}+\frac{r_{0}}{t} \cos \delta_{0}\right)\left(\frac{1}{2}|\Omega u|^{2}+\frac{1}{6}|u|^{6}\right) \\
& +\sqrt{2} \frac{r_{0}}{t}\left|\sin \delta_{0}\right| u_{\rho} u_{\alpha} .
\end{aligned}
$$




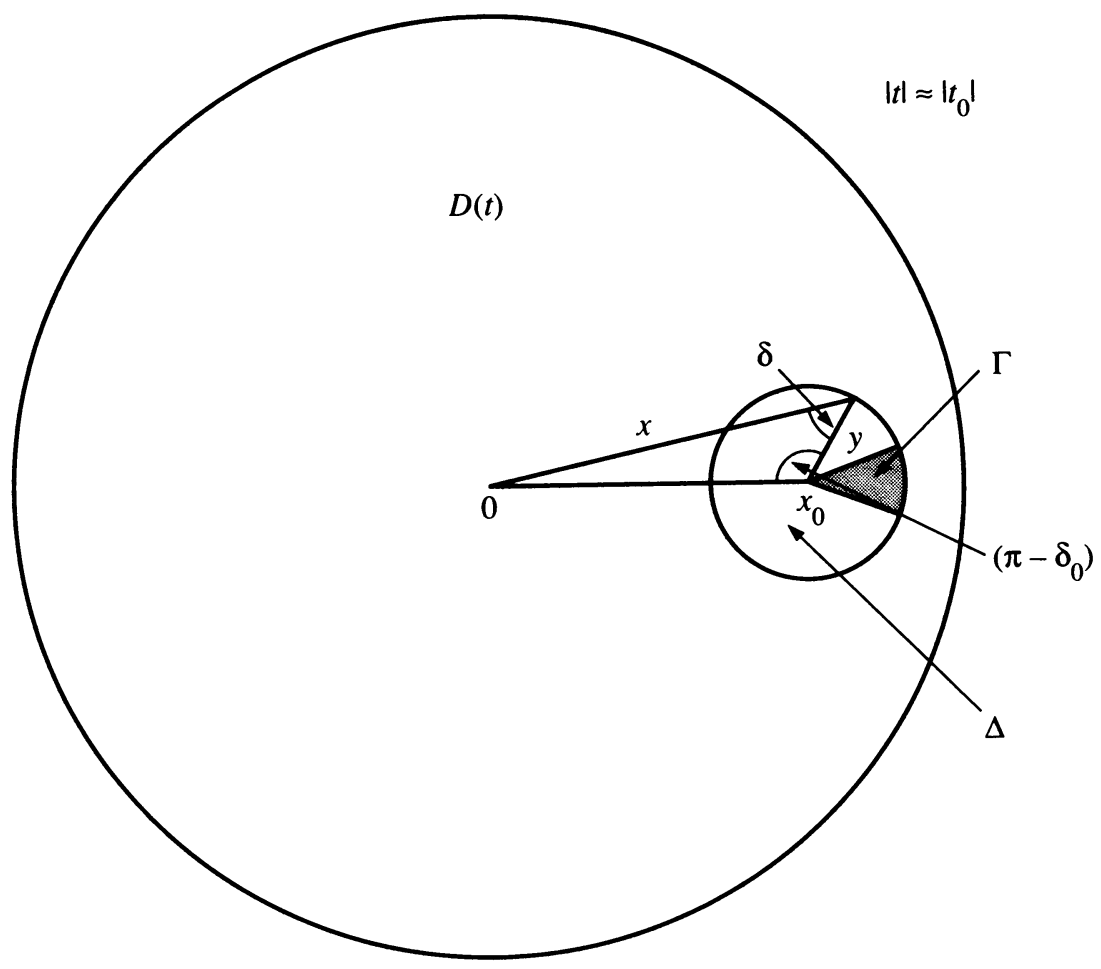

FiguRe 5

Estimating $|\Omega u| \geq\left|u_{\alpha}\right|$ as before, this is

$$
\begin{aligned}
\geq & \left(2-\frac{t_{0}-r_{0}}{t}\right)\left|u_{\rho}\right|^{2}-\frac{r_{0}}{2 t}\left(\sqrt{2} \sqrt{1+\cos \delta_{0}} u_{\rho}-\sqrt{1-\cos \delta_{0}} u_{\alpha}\right)^{2} \\
& +\frac{t_{0}}{t}\left(1+\frac{r_{0}}{t_{0}}\right) \frac{1}{2}\left|u_{\alpha}\right|^{2}+\frac{t_{0}}{t}\left(1+\frac{r_{0}}{t_{0}} \cos \delta_{0}\right) \frac{1}{6}|u|^{6} .
\end{aligned}
$$

Note that all the latter terms are nonnegative for $z \in M\left(z_{0}\right), z_{0} \in K$. By (4.14), and since $r_{0} \leq\left|t_{0}\right|$, for $t \leq 2 t_{0}$ we have $A_{0} \geq\left|u_{\rho}\right|^{2}$. Moreover, given $0<\varepsilon<1, z_{0} \in K$, let $t_{m} \leq 2 t_{0}<t_{m+1}$ and set

$$
\begin{aligned}
& \Gamma=\Gamma\left(\varepsilon ; z_{0}\right)=\left\{z \in M_{t_{m}}\left(z_{0}\right) ;\left|\delta_{0}\right| \leq \varepsilon^{1 / 4}\right\} \\
& \Delta=\Delta\left(\varepsilon ; z_{0}\right)=M_{t_{m}}\left(z_{0}\right) \backslash \Gamma .
\end{aligned}
$$

Note that by (4.13) on $\Gamma$ we can estimate

$$
\begin{aligned}
A_{0} & \geq\left|u_{\rho}\right|^{2}-\sqrt{2} \varepsilon^{1 / 4}\left|u_{\rho} u_{\alpha}\right| \\
& \geq\left|u_{\rho}\right|^{2}-\sqrt{2} \varepsilon^{1 / 4} d_{z_{0}}(u),
\end{aligned}
$$

while by (4.14) on $\Delta$ we have

$$
\begin{aligned}
A_{0} & \geq \frac{t_{0}}{t}\left(1+\frac{r_{0}}{t_{0}} \cos \delta_{0}\right) \frac{1}{6}|u|^{6} \geq \frac{1}{8}\left(1-\left(1-\frac{\varepsilon^{1 / 2}}{2}+\varepsilon\right)\right) \frac{1}{6}|u|^{6} \\
& \geq \frac{\varepsilon^{1 / 2}}{96}|u|^{6}-\varepsilon d_{z_{0}}(u) .
\end{aligned}
$$


Combining with (4.12) and Lemma 4.5, thus we obtain

$$
\begin{aligned}
\int_{\Gamma}\left|u_{\rho}\right|^{2} d o & \leq \int_{M_{t_{k}}\left(z_{0}\right)} A_{0} d o+\sqrt{2} \varepsilon^{1 / 4} E_{0} \\
& \leq\left(\varepsilon+\sqrt{2} \varepsilon^{1 / 4}\right) E_{0}+o(1)(1+\ln (1+C(\varepsilon))),
\end{aligned}
$$

(4.16) $\frac{\varepsilon^{1 / 2}}{96} \int_{\Delta}|u|^{6} d o \leq \int_{M_{t_{k}}\left(z_{0}\right)} A_{0} d o+\varepsilon E_{0} \leq 2 \varepsilon E_{0}+o(1)(1+\ln (1+C(\varepsilon)))$,

$$
\int_{M_{t_{l}}^{t_{m}\left(z_{0}\right)}}\left|u_{\rho}\right|^{2} d o \leq \int_{M_{t_{k}}^{t_{m}\left(z_{0}\right)}} A_{0} d o+\int_{M_{t_{l}}^{t_{k}\left(z_{0}\right)}}\left|u_{\rho}\right|^{2} d o \leq 2 \varepsilon E_{0}+o(1) \ln (1+C(\varepsilon)) \text {, }
$$

where $o(1) \rightarrow 0$ as $l \rightarrow \infty$. (We may assume $t_{l} \leq t_{k} \leq t_{m}$.)

Proof of Theorem 1.1. Given $\varepsilon>0$, we split the integral in (3.8) and use Hölder's inequality as follows

$$
\begin{aligned}
\int_{M_{t_{l}}\left(z_{0}\right)} \frac{|u|^{4}}{\left|t-t_{0}\right|} d o \leq & \int_{\Gamma}+\cdots+\int_{\Delta}+\cdots+\int_{M_{t_{l}}^{t_{m}\left(z_{0}\right)}}+\cdots \\
\leq & \left(\int_{\Gamma} \frac{|u|^{2}}{\left|t-t_{0}\right|^{2}} d o\right)^{1 / 2}\left(\int_{\Gamma}|u|^{6} d o\right)^{1 / 2} \\
& +\left(\int_{\Delta} \frac{|u|^{2}}{\left|t-t_{0}\right|^{2}} d o\right)^{1 / 2}\left(\int_{\Delta}|u|^{6} d o\right)^{1 / 2} \\
& +\left(\int_{M_{t_{l}}^{t_{m}\left(z_{0}\right)}} \frac{|u|^{2}}{\left|t-t_{0}\right|^{2}} d o\right)^{1 / 2}\left(\int_{M_{t_{l}}^{t_{m}\left(z_{0}\right)}}|u|^{6} d o\right)^{1 / 2} .
\end{aligned}
$$

By Lemma 3.1.(ii) and Lemma 3.2 this can be further estimated

$$
\begin{aligned}
\leq & \sqrt{6 E_{0}}\left(\int_{\Gamma} \frac{|u|^{2}}{\left|t-t_{0}\right|^{2}} d o\right)^{1 / 2}-C\left(E_{0}\right)\left(\int_{\Delta}|u|^{6} d o\right)^{1 / 2} \\
& +\sqrt{6 E_{0}}\left(\int_{M_{t_{l}}^{l_{l}\left(z_{0}\right)}} \frac{|u|^{2}}{\left|t-t_{0}\right|^{2}} d o\right)^{1 / 2} .
\end{aligned}
$$

By Lemma 3.1.(i) and (4.15)

$$
\begin{aligned}
\int_{\Gamma} \frac{|u|^{2}}{\left|t-t_{0}\right|^{2}} d o \leq & 4 \int_{\Gamma}\left|u_{\rho}\right|^{2} d o+2\left|t_{m}-t_{0}\right|^{-1} \int_{\partial D\left(t_{m} ; z_{0}\right)}|u|^{2} d o \\
\leq & 4\left(\varepsilon+\sqrt{2} \varepsilon^{1 / 4}\right) E_{0}+o(1)(1+\ln (1+C(\varepsilon))) \\
& +C\left(\int_{\partial D\left(t_{m} ; z_{0}\right)}|u|^{4} d o\right)^{1 / 2} .
\end{aligned}
$$

By Lemma 3.1.(iii) and Lemma 4.2 the latter

$$
\begin{aligned}
\int_{\partial D\left(t_{m}, z_{0}\right)}|u|^{4} d o & \leq C\left(\left(\int_{D\left(t_{m}\right)}|\nabla u|^{2} d x\right)^{1 / 2}+\left(\int_{D\left(t_{m}\right)}|u|^{6} d x\right)^{1 / 6}\right)\left(\int_{D\left(t_{m}\right)}|u|^{6} d x\right)^{1 / 2} \\
& \leq C\left(E_{0}\right) o(1),
\end{aligned}
$$


where $o(1) \rightarrow 0$ as $m \geq l$ tend to infinity. Similarly, by Lemma 3.1.(i), (iii), Lemma 4.2 and (4.17)

$$
\begin{aligned}
\int_{M_{t}^{t_{l}}\left(z_{0}\right)} \frac{|u|^{2}}{\left|t-t_{0}\right|^{2}} d o & \leq 4 \int_{M_{t_{l}}^{t_{m}\left(z_{0}\right)}}\left|u_{\rho}\right|^{2} d o+2\left|t_{l}-t_{0}\right|^{-1} \int_{\partial D\left(t_{l} ; z_{0}\right)}|u|^{2} d o \\
& \leq \varepsilon E_{0}+o(1)\left(C\left(E_{0}\right)+\ln (1+C(\varepsilon))\right) .
\end{aligned}
$$

Finally, by (4.16)

$$
\int_{\Delta}|u|^{6} d o \leq 192 \varepsilon^{1 / 2} E_{0}+o(1) \varepsilon^{-1 / 2}(1+\ln (1+C(\varepsilon))) .
$$

Hence, if we first choose $\varepsilon>0$ sufficiently small and then choose $l \in \mathbb{N}$ sufficiently large, the integral

$$
\int_{M_{t_{l}}\left(z_{0}\right)} \frac{u^{4}}{\left|t-t_{0}\right|} d o
$$

can be made as small as we please, uniformly in $z_{0} \in K_{t_{1}}$.

Remark. Since all error estimates are based on the qualitative statement (3.1), no a priori bounds for the solution $u$ on a cone $K$, depending only on $u_{0}, u_{1}$, and $K$, are obtained.

\section{A REMARK ON THE SUPER-CRITICAL CASE}

We add an observation on the super-critical case. Consider for simplicity the equation

$$
u_{t t}-\Delta u+u^{5}+u|u|^{p-2}=0 \text { in } \mathbb{R}^{3} \times[0, \infty[
$$

with initial data (1.2). (5.1) may be approximated by equations

$$
u_{t t}-\Delta u+u^{5}\left(1+\min \left\{|u|^{p-6}, k^{p-6}\right\}\right)=0 .
$$

By the preceding, (5.2) admits global $C^{2}$-solutions $u^{(k)}$; moreover, as in Rauch's Theorem 3.3 we may decompose

$$
u^{(k)}\left(z_{0}\right)=u^{(0)}\left(z_{0}\right)+v^{(k)}\left(z_{0}\right),
$$

where $u^{(0)}$ solves the homogenous wave equation with initial data $u_{0}, u_{1}$. Now, for suitable initial data, we obtain a uniform bound $\left|u^{(0)}(z)\right| \leq m_{0}$ for all $z \in$ $R^{3} \times\left[0, \infty\left[\right.\right.$; for instance, if $u_{0}, u_{1}$ have compact support. By (2.3), moreover, if

$$
\left|u^{(k)}\left(z_{0}\right)\right|=\sup _{z \in K_{0}\left(z_{0}\right)}\left|u^{(k)}(z)\right|=m_{k}
$$

we may estimate

$$
\begin{aligned}
m_{k} & =\left|u^{(k)}\left(z_{0}\right)\right| \leq\left|u^{(0)}\left(z_{0}\right)\right|+\left|v^{(k)}\left(z_{0}\right)\right| \\
& \leq m_{0}+\frac{m_{k}}{4 \pi} \int_{M_{0}\left(z_{0}\right)} \frac{\left|u^{(k)}\right|^{4}\left(1+\min \left\{\left|u^{(k)}\right|^{p-6}, k^{p-6}\right\}\right)}{t_{0}-t} d o \\
& \leq m_{0}+m_{k} \frac{1+k^{p-6}}{4 \pi} \int_{M_{0}\left(z_{0}\right)} \frac{\left|u^{(k)}\right|^{4}}{t_{0}-t} d o \\
& \leq m_{0}+C m_{k} k^{p-6}\left(E(u(0))+E^{2 / 3}(u(0))\right)<2 m_{0},
\end{aligned}
$$


if $k=2 m_{0}$ and if $E(u(0))$ is sufficiently small, depending on $m_{0}$, that is, on $u_{0}$ and $u_{1}$. Thus $u=u^{(k)}$ solves (5.1).

In particular, we obtain the following perturbation result:

Theorem 5.1. Suppose $u_{0} \in C^{3}\left(\mathbb{R}^{3}\right), u_{1} \in C^{2}\left(\mathbb{R}^{3}\right)$ have finite energy

$$
\int_{\mathbb{R}^{3}}\left(\frac{\left|u_{1}\right|^{2}+\left|\nabla u_{0}\right|^{2}}{2}+\frac{\left|u_{0}\right|^{6}}{6}\right) d x<\infty
$$

and suppose the solution $u^{(0)}$ to the homogeneous wave equation with initial data $u_{0}, u_{1}$ is uniformly bounded. Then there exists $\varepsilon_{0}>0$ such that for all $|\varepsilon|<\varepsilon_{0}$ the initial value problem for (5.1) with data $\varepsilon u_{0}, \varepsilon u_{1}$ admits a global $C^{2}$-solution.

However, "in the large" the super-critical case appears to be completely open.

\section{REFERENCES}

1. P. Brenner, On $L_{p}-L_{p^{\prime}}$ estimates for the wave equation, Math. Z. 145(1975), 251-254.

2. P. Brenner and W. von Wahl, Global classical solutions of non-linear wave equations, Math. Z. 176 (1981), 87-121.

3. F. E. Browder, On nonlinear wave equations, Math. Z. 80 (1962), 249-264.

4. J. Ginibre and G. Velo, The global Cauchy problem for the non-linear Klein-Gordon equation, Math. Z. 189 (1985), 487-505.

5. __ Scattering theory in the energy space for a large class of non-linear wave equations, Comm. Math. Phys. 123 (1989), 535-573.

6. M. G. Grillakis, Regularity and asymptotic behaviour of the wave equation with a critical nonlinearity, Ann. of Math. (2) (to appear).

7. F. John, Blow-up of solutions to nonlinear wave equations in three space dimensions, Manuscripta Math. 28 (1979), 235-268.

8. K. Jörgens, Das Anfangswertproblem im Grossen für eine Klasse nicht-linearer Wellengleichungen, Math. Z. 77 (1961), 295-308.

9. J. M. Lee and T. H. Parker, The Yamabe problem, Bull. Amer. Math. Soc. (N.S.) 17 (1987), $37-92$.

10. J.-L. Lions, Quelques méthodes de résolution des problèmes aux limites non linéaires, Dunod, Gauthier-Villars, Paris 1969.

11. H. Pecher, Ein nichtlinearer Interpolationssatz und seine Anwendung auf nichtlineare Wellengleichungen, Math. Z. 161 (1978), 9-40.

12. J. Rauch, The $u^{5}$-Klein-Gordon equation, (Brezis and Lions, eds.), Pitman Research Notes in Math., no. 53, pp. 335-364.

13. L. I. Schiff, Nonlinear meson theory of nuclear forces. I, Phys. Rev. 84 (1951), 1-9.

14. I. E. Segal, The global Cauchy problem for a relativistic scalar field with power interaction, Bull. Soc. Math. France 91 (1963), 129-135.

15. J. Shatah, Weak solutions and the development of singularities in the $S U(2)$ $\sigma$-model, Comm. Pure Appl. Math. 41 (1988), 459-469.

16. W. Strauss, Nonlinear wave equations, CBMS Lecture Notes, no. 73, Amer. Math. Soc., Providence, RI, 1989.

17. R. Strichartz, Restrictions of Fourier transforms to quadratic surfaces and decay of solutions of wave equations, Duke Math. J. 44 (1977), 705-714.

18. M. Struwe, Globally regular solutions to the $u^{5}$-Klein-Gordon equation, Ann. Sc. Norm. Sup. Pisa (Ser. 4) 15 (1988), 495-513. 
19. Y. Zheng, Concentration in sequences of solutions to the nonlinear Klein-Gordon equation, preprint, 1989.

20. L. V. Kapitanskii, The Cauchy problem for a semi-linear wave equation (1989).

21. J. Shatah and A. Tahvildar-Zadeh, Regularity of harmonic maps from Minkowski space into rotationally symmetric manifolds, Courant Institute, preprint, 1990.

Mathematik, ETH-Zentrum, CH-8092 Zürich, Switzerland 